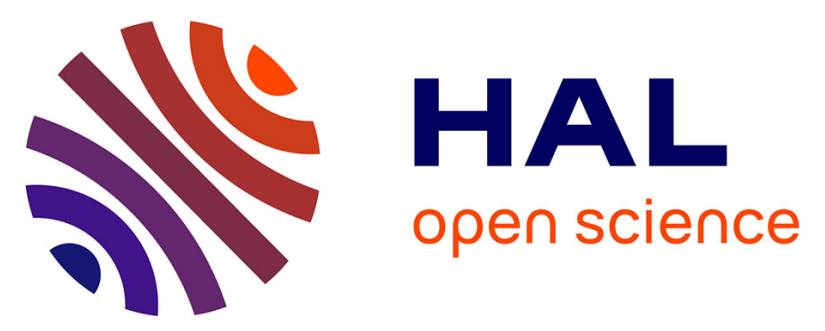

\title{
Effective resonant model and simulations in the time-domain of wave scattering from a periodic row of highly-contrasted inclusions
}

Marie Touboul, Kim Pham, Agnès Maurel, Jean-Jacques Marigo, Bruno Lombard, Cédric Bellis

\section{To cite this version:}

Marie Touboul, Kim Pham, Agnès Maurel, Jean-Jacques Marigo, Bruno Lombard, et al.. Effective resonant model and simulations in the time-domain of wave scattering from a periodic row of highlycontrasted inclusions. Journal of Elasticity, 2020, 142 (1), pp.53-82. hal-02625303v2

\section{HAL Id: hal-02625303 \\ https://hal.science/hal-02625303v2}

Submitted on 28 Jul 2020

HAL is a multi-disciplinary open access archive for the deposit and dissemination of scientific research documents, whether they are published or not. The documents may come from teaching and research institutions in France or abroad, or from public or private research centers.
L'archive ouverte pluridisciplinaire HAL, est destinée au dépôt et à la diffusion de documents scientifiques de niveau recherche, publiés ou non, émanant des établissements d'enseignement et de recherche français ou étrangers, des laboratoires publics ou privés. 


\title{
Effective resonant model and simulations in the time-domain of wave scattering from a periodic row of highly-contrasted inclusions
}

\author{
Marie Touboul ${ }^{\mathrm{a}}$, Kim Pham $^{\mathrm{b}, *}$, Agnès Maurel $^{\mathrm{c}}$, \\ Jean-Jacques Marigo ${ }^{\mathrm{d}}$, Bruno Lombard ${ }^{\mathrm{a}}$, Cédric Bellis ${ }^{\mathrm{a}}$ \\ ${ }^{a}$ Aix Marseille Univ, CNRS, Centrale Marseille, LMA UMR 7031, Marseille, France \\ ${ }^{b}$ IMSIA, CNRS, ENSTA ParisTech, 828 Bd des Maréchaux, 91732 Palaiseau, France \\ ${ }^{c}$ Institut Langevin, CNRS, ESPCI ParisTech, 1 rue Jussieu, 75005 Paris, France \\ ${ }^{d}$ Laboratoire de Mécanique des Solides, CNRS, Ecole Polytechnique, 91120 Palaiseau, France
}

\begin{abstract}
The time-domain propagation of scalar waves across a periodic row of inclusions is considered in 2D. As the typical wavelength within the background medium is assumed to be much larger than the spacing between inclusions and the row width, the physical configuration considered is in the low-frequency homogenization regime. Furthermore, a high contrast between one of the constitutive moduli of the inclusions and of the background medium is also assumed. So the wavelength within the inclusions is of the order of their typical size, which can further induce local resonances within the microstructure. In (20), two-scale homogenization techniques and matched-asymptotic expansions have been employed to derive, in the harmonic regime, effective jump conditions on an equivalent interface. This homogenized model is frequency-dependent due to the resonant behavior of the inclusions. In this context, the present article aims at investigating, directly in the time-domain, the scattering of waves by such a periodic row of resonant scatterers. Its effective behavior is first derived in the time-domain and some energy properties of the resulting homogenized model are analyzed. Time-domain numerical simulations are then performed to illustrate the main features of the effective interface model obtained and to assess its relevance in comparison with full-field simulations.
\end{abstract}

Keywords: dynamic homogenization, resonant media, matched asymptotic expansions

\section{Introduction}

Let us consider a microstructured medium composed of inclusions periodically embedded in a background medium, or matrix, and submitted to a dynamical excitation. When the characteristic wavelength within the matrix is much larger than the period length, the microstructure can be advantageously replaced, at the macro-scale, by a homogeneous effective medium. To do so, homogenization methods $(3 ; 21)$ can be employed to derive the properties of the sought effective medium. When the heterogeneities amount to a periodic row of inclusions (see Figure 1), then homogenization techniques and matched-asymptotic expansions can be deployed to obtain effective jump conditions on an equivalent interface, see $(15 ; 17 ; 8 ; 7 ; 16 ; 4)$. Furthermore, from energy-based considerations, its has been shown that an equivalent interface of non-zero width ensures the stability of the resulting model, while no field remains defined within the enlarged region. As a result, wave propagation across the

\footnotetext{
${ }^{*}$ Corresponding author. E-mail address: touboul@lma.cnrs-mrs.fr
} 
microstructured row of inclusions is modeled, in the long-wavelength regime, using effective jump conditions on both sides of an enlarged interface.

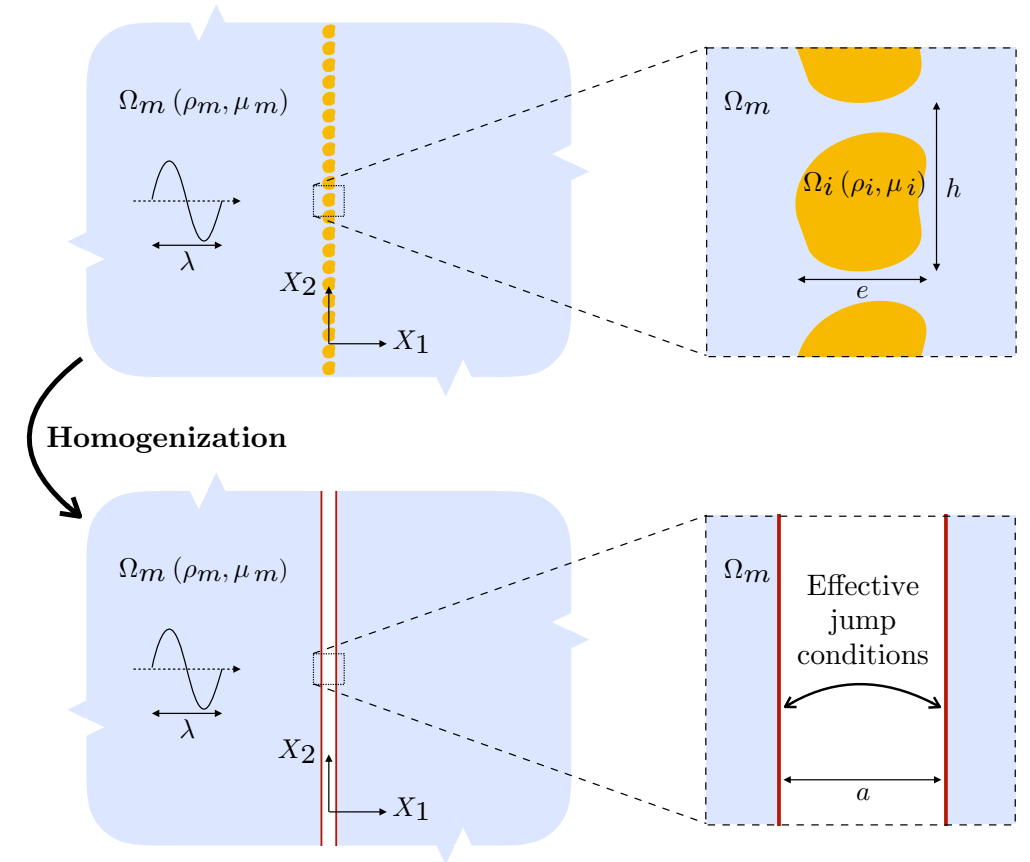

Figure 1: Homogenization process in the case of a single periodic row of inclusions.

For a periodic row of elastic inclusions, effective jump conditions have been derived in (16) in the case where the constitutive moduli of the inclusions and of the matrix are of the same order of magnitude. Nevertheless, when there is a high parameter contrast between the inclusions and the matrix then a specific homogenized model has to be formulated. Such a configuration is particularly relevant in the context of the metamaterials that are generally designed to exhibit specific effective behaviors, the control of waves being one application of interest in the dynamical context considered here. For microstructures characterized by high material contrasts then internal resonances can occur, a phenomenon which can be used in turn at the macroscopic level to drive the behavior of waves that propagate through them. As an example, resonant meta-surfaces have been designed to maximize sound absorption, see $(14 ; 22 ; 18)$. The homogenization of highly-contrasted materials has been studied in the volumic case $(26 ; 27 ; 1 ; 2 ; 9 ; 5 ; 24)$. The case of a periodic row of highly-contrasted inclusions has been investigated theoretically in (20) in the harmonic regime. This analysis leads to the construction of an equivalent enlarged interface on which a set of effective jump conditions applies. However, unlike the non-resonant case, the effective parameters of these conditions turn out to be frequency-dependent due to the local resonances that can occur within the microstructure.

The present paper focuses on the propagation of waves across a periodic row of highly-contrasted inclusions. The homogenization of such a resonant microstructure is specifically conducted in the transient regime. Effective properties are derived in the time domain in Section 2 and the resulting model is analyzed using energy-based arguments. In particular, a condition on the thickness of the enlarged interface is obtained to ensure the conservation of an energy. Section 3 aims at both illustrating the effective dynamical behavior obtained and at validating the effective model through comparisons with full-field, i.e. microstructure-based, simulations. One notes that the time-domain simulations presented here rely on the numerical method introduced in (25) to handle resonant jump 
conditions on meta-interfaces. Numerical results are discussed to highlight the computational merits of the homogenized model for the configuration of interest compared to full-field simulations. Results confirm a good agreement between full-field and homogenized simulations as the wavelength decreases towards the characteristic size of the inclusions.

\section{Homogenized resonant model in the time domain}

Let us consider the propagation of scalar waves in 2D across a periodic row of inclusions $\cup_{i} \Omega_{i}$ embedded within a homogeneous matrix $\Omega_{m}$, with both media being assumed to be isotropic. The thickness and the periodicity of the row are denoted by $e$ and $h$, respectively and we assume that $e=\mathcal{O}(h)$. The time and the spatial coordinates are denoted by $t$ and $\boldsymbol{X}=\left(X_{1}, X_{2}\right)$, respectively, with $X_{2}$ being the direction of periodicity of the inclusions as shown in Figure 1. The microstructured medium is characterized by two constitutive moduli, the mass density $\rho$ and the shear modulus $\mu$, that are piecewise constant:

$$
(\rho, \mu)(\boldsymbol{X})= \begin{cases}\left(\rho_{m}, \mu_{m}\right) & \text { in the matrix } \\ \left(\rho_{i}, \mu_{i}\right) & \text { in the inclusions. }\end{cases}
$$

The problem is considered within the framework of the linear anti-plane elasticity model. The time-domain governing equation for the scalar out-of-plane displacement $U$ writes:

$$
\operatorname{div}(\mu(\boldsymbol{X}) \nabla U(\boldsymbol{X}, t))=\rho(\boldsymbol{X}) \frac{\partial^{2} U}{\partial t^{2}}(\boldsymbol{X}, t) .
$$

Introducing the scalar velocity field $V=\partial U / \partial t$, this system can be rewritten as a first-order system in time for $V$ and for the stress vector $\Sigma=\left(\Sigma_{1}, \Sigma_{2}\right)^{T}$ :

$$
\left\{\begin{array}{l}
\frac{\partial \boldsymbol{\Sigma}}{\partial t}(\boldsymbol{X}, t)=\mu \boldsymbol{\nabla} V(\boldsymbol{X}, t) \\
\rho \frac{\partial V}{\partial t}(\boldsymbol{X}, t)=\operatorname{div} \boldsymbol{\Sigma}(\boldsymbol{X}, t)
\end{array}\right.
$$

with $V$ and $\boldsymbol{\Sigma} \cdot \boldsymbol{n}$ being continuous at each matrix/inclusion interface $\partial \Omega_{i}$, given that $\boldsymbol{n}$ is the inward unit normal on each $\partial \Omega_{i}$. The system (1) is also relevant to other physical phenomena, such as acoustic waves for which the fields $\Sigma, V, \rho$ and $1 / \mu$ would stand instead for velocity, pressure, compressibility and mass density, respectively.

Considering an illumination by an incident wave or external sources, a characteristic wavelength $\lambda$ within the matrix is assumed to be much larger than the spacing $h$ between successive inclusions. Defining the wavenumber within the matrix as $k_{m}=2 \pi / \lambda$, we introduce the ratio $\eta=k_{m} h$ that satisfies $\eta \ll 1$ for the configurations of interest. This geometrical assumption allows to homogenize the microstructure in the long-wavelength regime. The choice of a characteristic wavelength and the associated small parameter is discussed in Section 3.1.3. With high material contrast, the latter can be the seat of local resonances. Introducing the wavenumber $k_{i}=\omega \sqrt{\rho_{i} / \mu_{i}}$ within the inclusions, then such resonances can occur when the wavelength within an inclusion is of order of $h$, i.e. $k_{i} h=\mathcal{O}(1)$ $(2 ; 20)$. In particular, this assumption is met for a low contrast in mass density $\rho_{i} / \rho_{m}=\mathcal{O}(1)$ and a high contrast in shear modulus $\mu_{i} / \mu_{m}=\mathcal{O}\left(\eta^{2}\right)$, a configuration which we consider in the present study. These geometrical and material assumptions are summarized as follows:

Assumptions 1. The configuration satisfies $\eta=k_{m} h \ll 1$ while $\rho_{i} / \rho_{m}=\mathcal{O}(1)$ and $\mu_{i} / \mu_{m}=\mathcal{O}\left(\eta^{2}\right)$. 


\subsection{Effective jump conditions in the time domain}

New adimensionalized space-time coordinates are introduced to formulate the homogenization problem independently of the wavelength $\lambda$, i.e. one defines $\boldsymbol{x}=k_{m} \boldsymbol{X}$ and $\tau=k_{m} \sqrt{\frac{\mu_{m}}{\rho_{m}}} t$. One introduces the non-dimensionalized fields $v(\boldsymbol{x}, \tau)=\sqrt{\frac{\rho_{m}}{\mu_{m}}} V(\boldsymbol{X}, t)$ and $\boldsymbol{\sigma}(\boldsymbol{x}, \tau)=\frac{1}{\mu_{m}} \boldsymbol{\Sigma}(\boldsymbol{X}, t)$. The system (1) is then transformed into:

$$
\left\{\begin{array}{l}
\frac{\partial \boldsymbol{\sigma}}{\partial \tau}(\boldsymbol{x}, \tau)=\frac{\mu}{\mu_{m}} \nabla_{\boldsymbol{x}} v(\boldsymbol{x}, \tau), \\
\frac{\rho}{\rho_{m}} \frac{\partial v}{\partial \tau}(\boldsymbol{x}, \tau)=\operatorname{div}_{\boldsymbol{x}} \boldsymbol{\sigma}(\boldsymbol{x}, \tau),
\end{array}\right.
$$

while the continuity conditions on $v$ and $\boldsymbol{\sigma} \cdot \boldsymbol{n}$ at the matrix/inclusion interfaces are preserved.

\subsubsection{Matched asymptotic expansions}
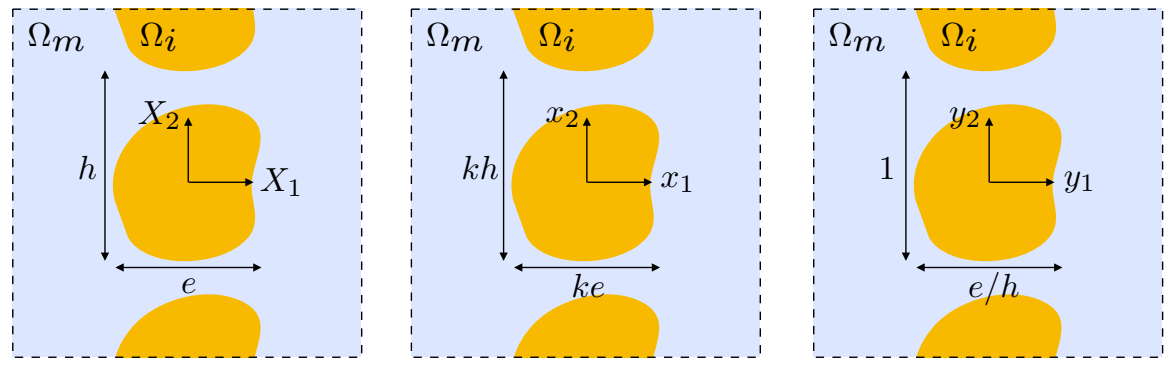

Figure 2: The different coordinate systems considered: (left) original coordinates, (center) adimensionalized coordinates, and (right) rescaled coordinates.

While the space coordinate $\boldsymbol{x}=\left(x_{1}, x_{2}\right)$ is appropriate to describe the slow variations of the wavefield, one introduces the rescaled coordinate $\boldsymbol{y}=\boldsymbol{x} / \eta$, see Figure 2 , which will be used to describe its small-scale fast variations. Note that, with a slight abuse of notation, the inclusion domain is referred to as $\cup_{i} \Omega_{i}$ in all three coordinate systems. Depending on the region of space considered, the fields $v$ and $\boldsymbol{\sigma}$ will be assumed to have specific dependencies on $\boldsymbol{x}$ and $\boldsymbol{y}$. In the far field, only the slow coordinate $\boldsymbol{x}$ is needed to describe the propagating field. Near the inclusions, dependence on the fast coordinate $\boldsymbol{y}$ is considered in order to describe field variations at the scale of the microstructure while slower variations along the interface are also accounted for through a dependence on $x_{2}$. Three regions are thus defined: an outer region (far field) for which $\boldsymbol{x} \in \mathbb{R}^{2}$, an inner region (near field) that excludes the inclusions, i.e. $\boldsymbol{y} \in \mathbb{R}^{2} \backslash \cup_{i} \Omega_{i}$ and $x_{2} \in \mathbb{R}$, and the inner region that coincides with the inclusions, i.e. $\boldsymbol{y} \in \cup_{i} \Omega_{i}$ and $x_{2} \in \mathbb{R}$. In these three regions, the velocity and stress fields are expanded using the following ansatz:

$$
\begin{gathered}
\begin{array}{c}
\text { Outer region } \\
\left(\boldsymbol{x} \in \mathbb{R}^{2}\right)
\end{array} \\
\left\{\begin{array}{c}
v=\sum_{j \geq 0} \eta^{j} v^{j}(\boldsymbol{x}, \tau) \\
\boldsymbol{\sigma}=\sum_{j \geq 0} \eta^{j} \boldsymbol{\sigma}^{j}(\boldsymbol{x}, \tau)
\end{array}\right.
\end{gathered}
$$

Inner region

$\left(\boldsymbol{y} \in \mathbb{R}^{2} \backslash \cup_{i} \Omega_{i}, x_{2} \in \mathbb{R}\right)$ $\left\{\begin{array}{l}v=\sum_{j \geq 0} \eta^{j} w^{j}\left(\boldsymbol{y}, x_{2}, \tau\right) \\ \boldsymbol{\sigma}=\sum_{j \geq 0} \eta^{j} \boldsymbol{s}^{j}\left(\boldsymbol{y}, x_{2}, \tau\right)\end{array}\right.$
Inclusions region

$$
\begin{gathered}
\left(\boldsymbol{y} \in \cup_{i} \Omega_{i}, x_{2} \in \mathbb{R}\right) \\
v=\sum_{j \geq 0} \eta^{j} w_{i}^{j}\left(\boldsymbol{y}, x_{2}, \tau\right) \\
\boldsymbol{\sigma}=\sum_{j \geq 0} \eta^{j} \boldsymbol{s}_{i}^{j}\left(\boldsymbol{y}, x_{2}, \tau\right) .
\end{gathered}
$$


The terms $\left(w^{j}, \boldsymbol{s}^{j}\right)_{j \geq 0}$ of the expansion of the solution $(v, \boldsymbol{\sigma})$ in the inner region are assumed to be periodic with respect to $y_{2}$. In addition, the continuity conditions on the inclusions interfaces read $w^{j}=w_{i}^{j}$ and $\boldsymbol{s}^{j} \cdot \boldsymbol{n}=\boldsymbol{s}_{i}^{j} \cdot \boldsymbol{n}$ on $\partial \Omega_{i}$. We consider the bounded domain described in $\boldsymbol{y}$-coordinates as $\Omega^{b}=\left[-y_{1}^{b}, y_{1}^{b}\right] \times[-1 / 2,1 / 2]$ with $y_{1}^{b}>e / h$, see Figure 3 . Later on, $\Omega_{i}$ will denote the inclusion in this bounded elementary cell. Moreover, one introduces the domain $\Omega=\lim _{y_{1}^{b} \rightarrow \pm \infty} \Omega^{b}=\mathbb{R} \times[-1 / 2,1 / 2]$ which is the elementary cell generating the periodic row of inclusions.

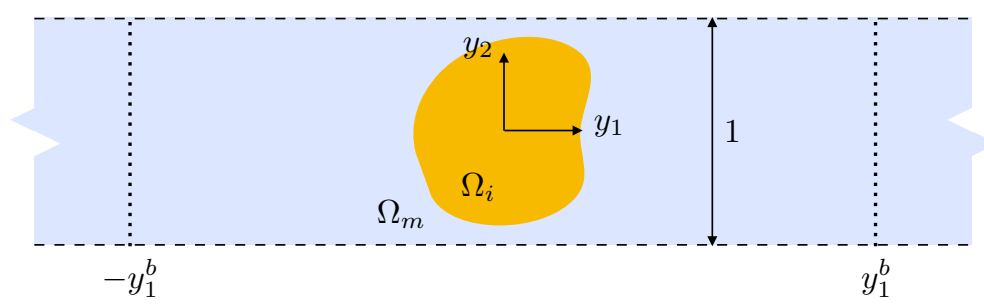

Figure 3: Bounded elementary cell $\Omega^{b}=\left[-y_{1}^{b}, y_{1}^{b}\right] \times\left[-\frac{1}{2}, \frac{1}{2}\right]$ with $y_{1}^{b}>\frac{e}{h}$.

As is customary in the two-scale asymptotic expansion approach, the differential operators featured in (2) are rewritten in the inner regions as:

$$
\boldsymbol{\nabla}_{\boldsymbol{x}} v \rightarrow \frac{1}{\eta} \nabla_{\boldsymbol{y}} v+\frac{\partial v}{\partial x_{2}} \boldsymbol{e}_{2} \quad \text { and } \quad \operatorname{div}_{\boldsymbol{x}} \boldsymbol{\sigma} \rightarrow \frac{1}{\eta} \operatorname{div}_{\boldsymbol{y}} \boldsymbol{\sigma}+\frac{\partial \boldsymbol{\sigma}}{\partial x_{2}} \cdot \boldsymbol{e}_{2}
$$

in terms of the canonical unit vectors $\boldsymbol{e}_{1}, \boldsymbol{e}_{2}$.

Based on Assumptions 1, we rewrite the parameter $\mu_{i}$ as $\mu_{i}=\eta^{2} \mu_{0}$ where $\mu_{0}$ is a reference modulus that satisfies $\mu_{0} / \mu_{m}=\mathcal{O}(1)$. Inserting (3) in (2) while using the previous differential operator identities, one obtains the following recursive differential problems at the orders $j \geq 0$ in the three regions:

$$
\left\{\begin{array}{l}
\frac{\partial \boldsymbol{\sigma}^{j}}{\partial \tau}=\nabla_{\boldsymbol{x}} v^{j} \\
\frac{\partial v^{j}}{\partial \tau}=\operatorname{div}_{\boldsymbol{x}} \boldsymbol{\sigma}^{j} \\
\frac{\partial \boldsymbol{s}^{j-1}}{\partial \tau}=\frac{\partial w^{j-1}}{\partial x_{2}} \boldsymbol{e}_{2}+\nabla_{\boldsymbol{y}} w^{j} \\
\frac{\partial w^{j-1}}{\partial \tau}=\frac{\partial \boldsymbol{s}^{j-1}}{\partial x_{2}} \cdot \boldsymbol{e}_{2}+\operatorname{div}_{\boldsymbol{y}} \boldsymbol{s}^{j}, \\
\frac{\partial \boldsymbol{s}_{i}^{j}}{\partial \tau}=\frac{\mu_{0}}{\mu_{m}}\left(\frac{\partial w_{i}^{j-2}}{\partial x_{2}} \boldsymbol{e}_{2}+\nabla_{\boldsymbol{y}} w_{i}^{j-1}\right) \\
\frac{\rho_{i}}{\rho_{m}} \frac{\partial w_{i}^{j-1}}{\partial \tau}=\frac{\partial \boldsymbol{s}_{i}^{j-1}}{\partial x_{2}} \cdot \boldsymbol{e}_{2}+\operatorname{div}_{\boldsymbol{y}} \boldsymbol{s}_{i}^{j},
\end{array}\right.
$$

where $\boldsymbol{s}^{p}, \boldsymbol{s}_{i}^{p}, w^{p}$ and $w_{i}^{p}$ vanish if $p<0$. Consequently, one can identify the $\mathcal{O}\left(\eta^{-1}\right)$-terms in the inner region:

$$
\left\{\begin{array}{c}
\nabla_{\boldsymbol{y}} w^{0}=\mathbf{0} \\
\operatorname{div}_{\boldsymbol{y}} \boldsymbol{s}^{0}=0 .
\end{array}\right.
$$


Then, in all three regions, the $\mathcal{O}(1)$-terms read

$$
\left\{\begin{array}{l}
\frac{\partial \boldsymbol{\sigma}^{0}}{\partial \tau}=\boldsymbol{\nabla}_{\boldsymbol{x}} v^{0} \\
\frac{\partial v^{0}}{\partial \tau}=\operatorname{div}_{\boldsymbol{x}} \boldsymbol{\sigma}^{0} \\
\frac{\partial \boldsymbol{s}^{0}}{\partial \tau}=\frac{\partial w^{0}}{\partial x_{2}} \boldsymbol{e}_{2}+\boldsymbol{\nabla}_{\boldsymbol{y}} w^{1}, \\
\frac{\partial w^{0}}{\partial \tau}=\frac{\partial \boldsymbol{s}^{0}}{\partial x_{2}} \cdot \boldsymbol{e}_{2}+\operatorname{div}_{\boldsymbol{y}} \boldsymbol{s}^{1}, \\
\frac{\partial \boldsymbol{s}_{i}^{0}}{\partial \tau}=\mathbf{0}, \\
\frac{\rho_{i}}{\rho_{m}} \frac{\partial w_{i}^{0}}{\partial \tau}=\frac{\partial \boldsymbol{s}_{i}^{0}}{\partial x_{2}} \cdot \boldsymbol{e}_{2}+\operatorname{div}_{\boldsymbol{y}} \boldsymbol{s}_{i}^{1}
\end{array}\right.
$$

One notes that the stress vector in the inclusion (6e) equals zero at the order $\mathcal{O}(1)$ whereas there is a contribution at the zeroth order for the stress vector in the matrix (6c). This is due to the high contrast in $\eta^{2}$ between $\mu_{m}$ and $\mu_{i}$ described in Assumption 1. Assuming that at the initial time $\boldsymbol{s}_{i}^{0}\left(\boldsymbol{y}, x_{2}, 0\right)=0$, then (6e) implies

$$
\boldsymbol{s}_{i}^{0}\left(\boldsymbol{y}, x_{2}, \tau\right)=0 \quad \forall \tau \geq 0 .
$$

Then the $\mathcal{O}(\eta)$-term within the inclusions reads

$$
\frac{\partial \boldsymbol{s}_{i}^{1}}{\partial \tau}=\frac{\mu_{0}}{\mu_{m}} \nabla_{\boldsymbol{y}} w_{i}^{0} .
$$

Finally, the outer and the inner solutions have to match in an intermediate region. Therefore, matching conditions are formulated in the limit $y_{1} \rightarrow \pm \infty$ for the near-field solution and $x_{1} \rightarrow 0^{ \pm}$for the far-field solution:

$$
\left\{\begin{array}{l}
v\left(0^{ \pm}, x_{2}, \tau\right):=\lim _{x_{1} \rightarrow 0^{ \pm}} v\left(x_{1}, x_{2}, \tau\right)=\lim _{y_{1} \rightarrow \pm \infty} w\left(\boldsymbol{y}, x_{2}, \tau\right), \\
\boldsymbol{\sigma}\left(0^{ \pm}, x_{2}, \tau\right):=\lim _{x_{1} \rightarrow 0^{ \pm}} \boldsymbol{\sigma}\left(x_{1}, x_{2}, \tau\right)=\lim _{y_{1} \rightarrow \pm \infty} \boldsymbol{s}\left(\boldsymbol{y}, x_{2}, \tau\right) .
\end{array}\right.
$$

The velocity and the stress vector in the outer and inner region are then expressed using the ansatz (3). Taylor expansions of the outer fields $v^{j}$ and $\boldsymbol{\sigma}^{j}$ at $x_{1}=0$ are written out for $j=0,1$ :

$$
\left\{\begin{aligned}
v^{j}\left(x_{1}, x_{2}, \tau\right) & =v^{j}\left(0^{ \pm}, x_{2}, \tau\right)+\eta y_{1} \partial_{x_{1}} v^{j}\left(0^{ \pm}, x_{2}, \tau\right) \\
\boldsymbol{\sigma}^{j}\left(x_{1}, x_{2}, \tau\right) & =\boldsymbol{\sigma}^{j}\left(0^{ \pm}, x_{2}, \tau\right)+\eta y_{1} \partial_{x_{1}} \boldsymbol{\sigma}^{j}\left(0^{ \pm}, x_{2}, \tau\right)
\end{aligned}\right.
$$

Eventually, the $\mathcal{O}(1)$-terms in (9) are identified:

$$
\left\{\begin{array}{l}
v^{0}\left(0^{ \pm}, x_{2}, \tau\right)=\lim _{y_{1} \rightarrow \pm \infty} w^{0}\left(\boldsymbol{y}, x_{2}, \tau\right) \\
\boldsymbol{\sigma}^{0}\left(0^{ \pm}, x_{2}, \tau\right)=\lim _{y_{1} \rightarrow \pm \infty} \boldsymbol{s}^{0}\left(\boldsymbol{y}, x_{2}, \tau\right)
\end{array}\right.
$$

and the $\mathcal{O}(\eta)$-terms give:

$$
\left\{\begin{array}{l}
v^{1}\left(0^{ \pm}, x_{2}, \tau\right)=\lim _{y_{1} \rightarrow \pm \infty}\left[w^{1}\left(\boldsymbol{y}, x_{2}, \tau\right)-y_{1} \frac{\partial v^{0}}{\partial x_{1}}\left(0^{ \pm}, x_{2}, \tau\right)\right], \\
\boldsymbol{\sigma}^{1}\left(0^{ \pm}, x_{2}, \tau\right)=\lim _{y_{1} \rightarrow \pm \infty}\left[\boldsymbol{s}^{1}\left(\boldsymbol{y}, x_{2}, \tau\right)-y_{1} \frac{\partial \boldsymbol{\sigma}^{0}}{\partial x_{1}}\left(0^{ \pm}, x_{2}, \tau\right)\right] .
\end{array}\right.
$$




\subsubsection{Jump conditions at the order $\mathcal{O}(1)$}

The homogenization of such a periodic row of highly-contrasted inclusions using matched asymptotic expansions has been investigated in (20) in the frequency domain. We follow the same method here and underline the differences due to the time-domain approach for the derivation of the jump conditions at the two first orders. Introducing for any function $f\left(x_{1}\right)$ the notation $\llbracket f \rrbracket=f\left(0^{+}\right)-f\left(0^{-}\right)$ then the jump condition for $v^{0}$ reads:

$$
\llbracket v^{0}\left(\cdot, x_{2}, \tau\right) \rrbracket=0
$$

as well as the jump condition for $\sigma_{1}^{0}$ :

$$
\llbracket \sigma_{1}^{0}\left(\cdot, x_{2}, \tau\right) \rrbracket=0
$$

Remark 1. We assume that $v^{0}$ and $\sigma_{1}^{0}$ are sufficiently smooth so that $\frac{\partial}{\partial \zeta} \llbracket v^{0}\left(\cdot, x_{2}, \tau\right) \rrbracket=\llbracket \frac{\partial}{\partial \zeta} v^{0}\left(\cdot, x_{2}, \tau\right) \rrbracket$ and $\frac{\partial}{\partial \zeta} \llbracket \sigma_{1}^{0}\left(\cdot, x_{2}, \tau\right) \rrbracket=\llbracket \frac{\partial}{\partial \zeta} \sigma_{1}^{0}\left(\cdot, x_{2}, \tau\right) \rrbracket$ for $\zeta=x_{2}$ or $\zeta=\tau$. Hence equations (13) and (14) imply:

$$
\llbracket \frac{\partial}{\partial \zeta} v^{0}\left(\cdot, x_{2}, \tau\right) \rrbracket=0
$$

and

$$
\llbracket \frac{\partial}{\partial \zeta} \sigma_{1}^{0}\left(\cdot, x_{2}, \tau\right) \rrbracket=0
$$

for $\zeta=x_{2}$ or $\tau$.

More precisely, for the zeroth order velocity field we have:

$$
v^{0}\left(0^{ \pm}, x_{2}, \tau\right)=w^{0}\left(x_{2}, \tau\right) .
$$

\subsubsection{Jump conditions at the order $\mathcal{O}(\eta)$}

Jump condition for $v^{1}$. Due to the continuity condition (16) with $\zeta=\tau$ and based on (6a), we get that $\partial v^{0} / \partial x_{1}$ is continuous at $x_{1}=0$. Thus, from (12a), one obtains the jump condition:

$$
\llbracket v^{1}\left(\cdot, x_{2}, \tau\right) \rrbracket=\lim _{y_{1} \rightarrow+\infty}\left[w^{1}\left(y_{1}, y_{2}, x_{2}, \tau\right)-w^{1}\left(-y_{1}, y_{2}, x_{2}, \tau\right)-2 y_{1} \frac{\partial v^{0}}{\partial x_{1}}\left(0, x_{2}, \tau\right)\right] .
$$

At this point, an expression for $w_{1}$ turns out to be needed. To get one, we combine (5b), (6c), (17), (11b), (6a) and the fact that $\partial \boldsymbol{s}^{0} / \partial \tau \cdot \boldsymbol{n}=\partial \boldsymbol{s}_{i}^{0} / \partial \tau \cdot \boldsymbol{n}=0$ on $\partial \Omega_{i}$ due to (6e) to obtain that the field $w^{1}$ is part of the following problem:

$$
\begin{cases}\operatorname{div}_{\boldsymbol{y}} \frac{\partial \boldsymbol{s}^{0}}{\partial \tau}=0 & \text { in } \Omega \backslash \Omega_{i}, \\ \frac{\partial \boldsymbol{s}^{0}}{\partial \tau}=\frac{\partial v^{0}}{\partial x_{2}}\left(0, x_{2}, \tau\right) \boldsymbol{e}_{2}+\boldsymbol{\nabla}_{\boldsymbol{y}} w^{1} & \text { in } \Omega \backslash \Omega_{i} \\ \frac{\partial \boldsymbol{s}^{0}}{\partial \tau} \cdot \boldsymbol{n}=0 & \text { on } \partial \Omega_{i}, \\ \lim _{y_{1} \rightarrow \pm \infty} \frac{\partial \boldsymbol{s}^{0}}{\partial \tau}=\boldsymbol{\nabla}_{\boldsymbol{x}} v^{0}\left(0, x_{2}, \tau\right) . & \end{cases}
$$


The problem above being linear with respect to $\partial v^{0} / \partial x_{1}\left(0, x_{2}, \tau\right)$ and $\partial v^{0} / \partial x_{2}\left(0, x_{2}, \tau\right)$, the field $w^{1}$ can be written as

$$
w^{1}\left(\boldsymbol{y}, x_{2}, \tau\right)=\frac{\partial v^{0}}{\partial x_{1}}\left(0, x_{2}, \tau\right)\left[\Phi^{(1)}(\boldsymbol{y})+y_{1}\right]+\frac{\partial v^{0}}{\partial x_{2}}\left(0, x_{2}, \tau\right) \Phi^{(2)}(\boldsymbol{y})+\tilde{w}\left(x_{2}, \tau\right),
$$

where for $j=1,2$ the fields $\Phi^{(j)}$ are $y_{2}$-periodic and solutions of:

$$
\begin{cases}\Delta_{\boldsymbol{y}} \Phi^{(j)}(\boldsymbol{y})=0 & \text { in } \Omega \backslash \Omega_{i} \\ {\left[\boldsymbol{\nabla}_{\boldsymbol{y}} \Phi^{(j)}(\boldsymbol{y})+\boldsymbol{e}_{j}\right] \cdot \boldsymbol{n}=0} & \text { on } \partial \Omega_{i} \\ \lim _{y_{1} \rightarrow \pm \infty} \boldsymbol{\nabla}_{\boldsymbol{y}} \Phi^{(j)}\left(y_{1}, y_{2}\right)=\mathbf{0} & \end{cases}
$$

Owing to the third equation in (21), some constants $B_{j}$ are introduced for $j=1,2$ as

$$
B_{j}=\lim _{y_{1} \rightarrow+\infty}\left[\Phi^{(j)}\left(y_{1}, y_{2}\right)-\Phi^{(j)}\left(-y_{1}, y_{2}\right)\right]
$$

Finally, inserting (20) in (18) leads to

$$
\llbracket v^{1}\left(\cdot, x_{2}, \tau\right) \rrbracket=B_{1} \frac{\partial v^{0}}{\partial x_{1}}\left(0, x_{2}, \tau\right)+B_{2} \frac{\partial v^{0}}{\partial x_{2}}\left(0, x_{2}, \tau\right) .
$$

Jump condition for $\sigma_{1}^{1}$. Using both (15) and (16) with $\zeta=x_{2}$ and $\zeta=\tau$ together with (6a) and (6b) implies that $\frac{\partial}{\partial x_{1}} \sigma_{1}^{0}\left(\cdot, x_{2}, \tau\right)$ is continuous at $x_{1}=0$. The equation (12b) integrated along $y_{2} \in[-1 / 2,1 / 2]$ provides the jump condition for $\sigma_{1}^{1}$ :

$$
\llbracket \sigma_{1}^{1}\left(\cdot, x_{2}, \tau\right) \rrbracket=\lim _{y_{1}^{b} \rightarrow+\infty} \int_{-\frac{1}{2}}^{\frac{1}{2}}\left[s_{1}^{1}\left(y_{1}^{b}, y_{2}, x_{2}, \tau\right)-s_{1}^{1}\left(-y_{1}^{b}, y_{2}, x_{2}, \tau\right)\right] \mathrm{d} y_{2}-2 y_{1}^{b} \frac{\partial \sigma_{1}^{0}}{\partial x_{1}}\left(0, x_{2}, \tau\right) .
$$

We start with (6d) integrated on $\Omega^{b} \backslash \Omega_{i}$ :

$$
\underbrace{\int_{\Omega^{b} \backslash \Omega_{i}} \frac{\partial w^{0}}{\partial \tau} \mathrm{d} \boldsymbol{y}}_{I_{1}}-\underbrace{-\int_{\Omega^{b} \backslash \Omega_{i}} \frac{\partial \boldsymbol{s}^{0}}{\partial x_{2}} \cdot \boldsymbol{e}_{2} \mathrm{~d} \boldsymbol{y}}_{I_{2}} \underbrace{-\int_{\Omega^{b} \backslash \Omega_{i}} \operatorname{div}_{\boldsymbol{y}} \boldsymbol{s}^{1} \mathrm{~d} \boldsymbol{y}}_{I_{3}}=0 .
$$

We introduce the parameter $\varphi$ satisfying $0<\varphi<1$ and such that $S_{i}=\frac{e \varphi}{h}$ is the surface of the inclusion. The first integral $I_{1}$ can be computed:

$$
I_{1}=\frac{\partial v^{0}}{\partial \tau}\left(0, x_{2}, \tau\right)\left[2 y_{1}^{b}-\frac{e \varphi}{h}\right]
$$

The derivative $\frac{\partial}{\partial \tau} s_{2}^{0}\left(\boldsymbol{y}, x_{2}, \tau\right)$ can be expressed thanks to the cell functions defined in (21) using the second equation of (19) together with (20) and (6a) :

$$
\frac{\partial s_{2}^{0}}{\partial \tau}\left(\boldsymbol{y}, x_{2}, \tau\right)=\frac{\partial \sigma_{1}^{0}}{\partial \tau}\left(0, x_{2}, \tau\right) \frac{\partial \Phi^{(1)}}{\partial y_{2}}(\boldsymbol{y})+\frac{\partial \sigma_{2}^{0}}{\partial \tau}\left(0, x_{2}, \tau\right)\left[\frac{\partial \Phi^{(2)}}{\partial y_{2}}(\boldsymbol{y})+1\right] .
$$

Integrating (25) in time and differentiating it with respect to $x_{2}$, the second integral $I_{2}$ writes:

$$
I_{2}=-\frac{\partial \sigma_{1}^{0}}{\partial x_{2}}\left(0, x_{2}, \tau\right) \int_{\Omega^{b} \backslash \Omega_{i}} \frac{\partial \Phi^{(1)}}{\partial y_{2}}(\boldsymbol{y}) \mathrm{d} \boldsymbol{y}-\frac{\partial \sigma_{2}^{0}}{\partial x_{2}}\left(0, x_{2}, \tau\right)\left[\int_{\Omega^{b} \backslash \Omega_{i}} \frac{\partial \Phi^{(2)}}{\partial y_{2}}(\boldsymbol{y}) \mathrm{d} \boldsymbol{y}+2 y_{1}^{b}-\frac{e \varphi}{h}\right]
$$


Owing to the periodicity of $\boldsymbol{s}^{1}$ with respect to $y_{2}$ and introducing the curvilinear abscissa $\ell$ on $\partial \Omega_{i}$, with $\boldsymbol{n}$ being the associated inward unit normal, then $I_{3}$ can be expressed as

$$
I_{3}=-\int_{-\frac{1}{2}}^{\frac{1}{2}}\left[s_{1}^{1}\left(y_{1}^{b}, y_{2}, x_{2}, \tau\right)-s_{1}^{1}\left(-y_{1}^{b}, y_{2}, x_{2}, \tau\right)\right] \mathrm{d} y_{2}-\int_{\partial \Omega_{i}} \boldsymbol{s}^{1} \cdot \boldsymbol{n} \mathrm{d} \ell .
$$

Due to the continuity condition $\boldsymbol{s}^{1} \cdot \boldsymbol{n}=\boldsymbol{s}_{i}^{1} \cdot \boldsymbol{n}$ on $\partial \Omega_{i}$, the second term in $I_{3}$ can be recast as

$$
-\int_{\partial \Omega_{i}} \boldsymbol{s}^{1} \cdot \boldsymbol{n} \mathrm{d} \ell=\int_{\Omega_{i}} \operatorname{div}_{\boldsymbol{y}} \boldsymbol{s}_{i}^{1} \mathrm{~d} \boldsymbol{y}
$$

We now use (6f) together with (7) and (8). For the boundary condition, the continuity condition $w^{j}=w_{i}^{j}$ on $\partial \Omega_{i}$ and (5a) with (11a) is used. Then, the field $\boldsymbol{s}_{i}^{1}$ satisfies

$$
\begin{cases}\frac{\partial \boldsymbol{s}_{i}^{1}}{\partial \tau}\left(\boldsymbol{y}, x_{2}, \tau\right)=\frac{\mu_{0}}{\mu_{m}} \nabla_{\boldsymbol{y}} w_{i}^{0}\left(\boldsymbol{y}, x_{2}, \tau\right) & \left(\boldsymbol{y} \in \Omega_{i}\right), \\ \frac{\rho_{i}}{\rho_{m}} \frac{\partial w_{i}^{0}}{\partial \tau}\left(\boldsymbol{y}, x_{2}, \tau\right)=\operatorname{div}_{\boldsymbol{y}} \boldsymbol{s}_{i}^{1}\left(\boldsymbol{y}, x_{2}, \tau\right) & \left(\boldsymbol{y} \in \Omega_{i}\right), \\ w_{i}^{0}\left(\boldsymbol{y}, x_{2}, \tau\right)=v^{0}\left(0, x_{2}, \tau\right) & \left(\boldsymbol{y} \in \partial \Omega_{i}\right) .\end{cases}
$$

Therefore, one concludes that the field $w_{i}^{0}$ is solution of

$$
\begin{cases}\frac{\rho_{i}}{\rho_{m}} \frac{\partial^{2} w_{i}^{0}}{\partial \tau^{2}}\left(\boldsymbol{y}, x_{2}, \tau\right)-\frac{\mu_{0}}{\mu_{m}} \Delta_{\boldsymbol{y}} w_{i}^{0}\left(\boldsymbol{y}, x_{2}, \tau\right)=0 & \left(\boldsymbol{y} \in \Omega_{i}\right) \\ w_{i}^{0}\left(\boldsymbol{y}, x_{2}, \tau\right)=v^{0}\left(0, x_{2}, \tau\right) & \left(\boldsymbol{y} \in \partial \Omega_{i}\right)\end{cases}
$$

and one can rewrite

$$
I_{3}=-\int_{-\frac{1}{2}}^{\frac{1}{2}}\left[s_{1}^{1}\left(y_{1}^{b}, y_{2}, x_{2}, \tau\right)-s_{1}^{1}\left(-y_{1}^{b}, y_{2}, x_{2}, \tau\right)\right] \mathrm{d} y_{2}+\frac{\rho_{i}}{\rho_{m}} \int_{\Omega_{i}} \frac{\partial w_{i}^{0}}{\partial \tau} \mathrm{d} \boldsymbol{y} .
$$

Collecting the three integrals (24), (26) and (28) and using (6b) one gets

$$
\begin{aligned}
& \int_{-\frac{1}{2}}^{\frac{1}{2}}\left[s_{1}^{1}\left(y_{1}^{b}, y_{2}, x_{2}, \tau\right)-s_{1}^{1}\left(-y_{1}^{b}, y_{2}, x_{2}, \tau\right)\right] \mathrm{d} y_{2}-2 y_{1}^{b} \frac{\partial \sigma_{1}^{0}}{\partial x_{1}}\left(0, x_{2}, \tau\right)=-\frac{e \varphi}{h} \frac{\partial \sigma_{1}^{0}}{\partial x_{1}}\left(0, x_{2}, \tau\right) \\
& -\frac{\partial \sigma_{1}^{0}}{\partial x_{2}}\left(0, x_{2}, \tau\right) \int_{\Omega^{b} \backslash \Omega_{i}} \frac{\partial \Phi^{(1)}}{\partial y_{2}}(\boldsymbol{y}) \mathrm{d} \boldsymbol{y}-\frac{\partial \sigma_{2}^{0}}{\partial x_{2}}\left(0, x_{2}, \tau\right) \int_{\Omega^{b} \backslash \Omega_{i}} \frac{\partial \Phi^{(2)}}{\partial y_{2}}(\boldsymbol{y}) \mathrm{d} \boldsymbol{y}+\frac{\rho_{i}}{\rho_{m}} \int_{\Omega_{i}} \frac{\partial w_{i}^{0}}{\partial \tau} \mathrm{d} \boldsymbol{y} .
\end{aligned}
$$

Introducing the following two parameters

$$
C_{j}=-\int_{\Omega \backslash \Omega_{i}} \frac{\partial \Phi^{(j)}}{\partial y_{2}}(\boldsymbol{y}) \mathrm{d} \boldsymbol{y} \quad \text { for } j=1,2
$$

and taking the limit $y_{1}^{b} \rightarrow+\infty$ in (29) finally entails the following jump condition

$$
\llbracket \sigma_{1}^{1}\left(\cdot, x_{2}, \tau\right) \rrbracket=-\frac{e \varphi}{h} \frac{\partial \sigma_{1}^{0}}{\partial x_{1}}\left(0, x_{2}, \tau\right)+C_{1} \frac{\partial \sigma_{1}^{0}}{\partial x_{2}}\left(0, x_{2}, \tau\right)+C_{2} \frac{\partial \sigma_{2}^{0}}{\partial x_{2}}\left(0, x_{2}, \tau\right)+\frac{\rho_{i}}{\rho_{m}} \int_{\Omega_{i}} \frac{\partial w_{i}^{0}}{\partial \tau} \mathrm{d} \boldsymbol{y}
$$




\subsubsection{Final effective jump conditions}

The jump conditions derived in the previous sections are associated with an interface of zero thickness as one considers jumps in the limit $x_{1} \rightarrow 0^{ \pm}$. Yet, it has been shown in (15) and (16) that a non-zero thickness $a$ allows to define an interface energy, which would in turn ensure the stability of the effective model. Using such an energy-based argument, we will justify in the next section that an enlarged interface is also required in the resonant case considered here. Meanwhile, the present section establishes the final effective jump conditions expressed relatively to an enlarged interface of thickness $a>0$ in the original system of space coordinates with $a / h=\mathcal{O}(1)$. The effective jump conditions thus obtained are equivalent up to order $\mathcal{O}\left(\eta^{2}\right)$ to the ones formulated in Section 2.1.3.

For any function $f\left(x_{1}\right)$, we define the jump and the mean value around the (centered) enlarged interface of thickness $a$ :

$$
\llbracket f \rrbracket_{k_{m} a}=f\left(k_{m} a / 2\right)-f\left(-k_{m} a / 2\right) \quad \text { and } \quad\langle f\rangle_{k_{m} a}=\frac{1}{2}\left(f\left(k_{m} a / 2\right)+f\left(-k_{m} a / 2\right)\right) .
$$

Setting $f=v\left(\cdot, x_{2}, \tau\right)$ or $f=\sigma_{1}\left(\cdot, x_{2}, \tau\right)$, we seek asymptotics of the form:

$$
\llbracket f \rrbracket_{k_{m} a}=\llbracket f^{0} \rrbracket_{k_{m} a}+\eta \llbracket f^{1} \rrbracket_{k_{m} a}+\mathcal{O}\left(\eta^{2}\right) .
$$

To do so, we consider the following Taylor expansions between $0^{ \pm}$and $\pm k_{m} a / 2$ for the function $f$ defined above

$$
\left\{\begin{array}{l}
\llbracket f^{0} \rrbracket=\llbracket f^{0} \rrbracket_{k_{m} a}-\eta \frac{a}{h}\left\langle\frac{\partial f^{0}}{\partial x_{1}}\right\rangle_{k_{m} a}+\mathcal{O}\left(\eta^{2}\right) \\
\llbracket f^{1} \rrbracket=\llbracket f^{1} \rrbracket_{k_{m} a}+\mathcal{O}(\eta)
\end{array}\right.
$$

Likewise for $g=\partial v^{0} / \partial x_{j}\left(\cdot, x_{2}, \tau\right)$ or $g=\partial \sigma_{k}^{0} / \partial x_{j}\left(\cdot, x_{2}, \tau\right)$ with $j, k=1,2$ one considers

$$
g(0)=\langle g\rangle_{k_{m} a}+\mathcal{O}(\eta)
$$

Due to (34), the solution to (27) is expanded as $w_{i}^{0}=w_{i}+\mathcal{O}(\eta)$ where the field $w_{i}$ is solution to the inner problem

$$
\begin{cases}\frac{\rho_{i}}{\rho_{m}} \frac{\partial^{2} w_{i}}{\partial \tau^{2}}\left(\boldsymbol{y}, x_{2}, \tau\right)-\frac{\mu_{0}}{\mu_{m}} \Delta_{\boldsymbol{y}} w_{i}\left(\boldsymbol{y}, x_{2}, \tau\right)=0 & \left(\boldsymbol{y} \in \Omega_{i}\right), \\ w_{i}\left(\boldsymbol{y}, x_{2}, \tau\right)=\left\langle v\left(\cdot, x_{2}, \tau\right)\right\rangle_{k_{m} a} & \left(\boldsymbol{y} \in \partial \Omega_{i}\right) .\end{cases}
$$

Introducing the following coefficients

$$
S=\frac{a}{h}-\frac{e \varphi}{h} \quad \text { and } \quad B=\frac{a}{h}+B_{1}
$$

then combining the equations (32-34), (13), (23), (14), (31) and (35), we get

$$
\left\{\begin{array}{l}
\llbracket v \rrbracket_{k_{m} a}=\eta\left\{B\left\langle\frac{\partial v}{\partial x_{1}}\right\rangle_{k_{m} a}+B_{2}\left\langle\frac{\partial v}{\partial x_{2}}\right\rangle_{k_{m} a}\right\}+\mathcal{O}\left(\eta^{2}\right), \\
\llbracket \sigma_{1} \rrbracket_{k_{m} a}=\eta\left\{S\left\langle\frac{\partial \sigma_{1}}{\partial x_{1}}\right\rangle_{k_{m} a}+C_{1}\left\langle\frac{\partial \sigma_{1}}{\partial x_{2}}\right\rangle_{k_{m} a}+C_{2}\left\langle\frac{\partial \sigma_{2}}{\partial x_{2}}\right\rangle_{k_{m} a}+\frac{\rho_{i}}{\rho_{m}} \int_{\Omega_{i}} \frac{\partial w_{i}}{\partial \tau} \mathrm{d} \boldsymbol{y}\right\}+\mathcal{O}\left(\eta^{2}\right) .
\end{array}\right.
$$

We now consider the associated approximation of the fields, as $\left(v^{h}, \boldsymbol{\sigma}^{h}\right)=\left(v^{0}+\eta v^{1}, \boldsymbol{\sigma}^{0}+\eta \boldsymbol{\sigma}^{1}\right)$, which are solution to (2) in the matrix domain while satisfying the jump conditions above but truncated at 
the second order. These fields are then transposed in the original coordinate system $(\boldsymbol{X}, t)$, see Figure 2 , as $\left(V^{h}, \Sigma^{h}\right)$, to get finally the following effective model at order $\mathcal{O}(\eta)$ :

$$
\left\{\begin{array}{lr}
\frac{\partial \boldsymbol{\Sigma}^{h}}{\partial t}=\mu_{m} \boldsymbol{\nabla} V^{h} & \left(\left|X_{1}\right| \geq a / 2, X_{2} \in \mathbb{R}\right) \\
\rho_{m} \frac{\partial V^{h}}{\partial t}=\operatorname{div} \boldsymbol{\Sigma}^{h} & \left(\left|X_{1}\right| \geq a / 2, X_{2} \in \mathbb{R}\right) \\
\llbracket V^{h} \rrbracket_{a}=h\left\{B\left\langle\frac{\partial V^{h}}{\partial X_{1}}\right\rangle_{a}+B_{2}\left\langle\frac{\partial V^{h}}{\partial X_{2}}\right\rangle_{a}\right\} & \left(X_{2} \in \mathbb{R}\right), \\
\llbracket \Sigma_{1}^{h} \rrbracket_{a}=h\left\{S\left\langle\frac{\partial \Sigma_{1}^{h}}{\partial X_{1}}\right\rangle_{a}+C_{1}\left\langle\frac{\partial \Sigma_{1}^{h}}{\partial X_{2}}\right\rangle_{a}+C_{2}\left\langle\frac{\partial \Sigma_{2}^{h}}{\partial X_{2}}\right\rangle_{a}+\rho_{i} \int_{\Omega_{i}} \frac{\partial W_{i}}{\partial t} \mathrm{~d} \boldsymbol{y}\right\} & \left(X_{2} \in \mathbb{R}\right) .
\end{array}\right.
$$

In (37), $W_{i}\left(\boldsymbol{y}, X_{2}, t\right)=\sqrt{\frac{\mu_{m}}{\rho_{m}}} w_{i}\left(\boldsymbol{y}, x_{2}, \tau\right)$ so that $W_{i}$ is solution of

$$
\begin{cases}\frac{\partial \boldsymbol{\Sigma}_{i}}{\partial t}\left(\boldsymbol{y}, X_{2}, t\right)=\frac{\mu_{i}}{h} \boldsymbol{\nabla}_{\boldsymbol{y}} W_{i}\left(\boldsymbol{y}, X_{2}, t\right) & \left(\boldsymbol{y} \in \Omega_{i}\right), \\ \rho_{i} \frac{\partial W_{i}}{\partial t}\left(\boldsymbol{y}, X_{2}, t\right)=\frac{1}{h} \operatorname{div}_{\boldsymbol{y}} \boldsymbol{\Sigma}_{i}\left(\boldsymbol{y}, X_{2}, t\right) & \left(\boldsymbol{y} \in \Omega_{i}\right), \\ W_{i}\left(\boldsymbol{y}, X_{2}, t\right)=\left\langle V^{h}\left(\cdot, X_{2}, t\right)\right\rangle_{a} & \left(\boldsymbol{y} \in \partial \Omega_{i}\right) .\end{cases}
$$

One notices that the field $W_{i}$ is a function of $X_{2}$ through the prescribed boundary condition $\left\langle V^{h}\left(\cdot, X_{2}, t\right)\right\rangle_{a}$. The five effective parameters $B, B_{2}, S, C_{1}$ and $C_{2}$ are given by (36), (22), and (30).

Remark 2. When $\rho_{i}=0$, as it is in the case when the inclusions are replaced by voids, the resonant term $\rho_{i} \int_{\Omega_{i}} \frac{\partial W_{i}}{\partial t} \mathrm{~d} \boldsymbol{y}$ in (37) vanishes. Therefore, when the contribution of the resonances is not activated, one recovers the model of (15).

Remark 3. The equivalence between this model and the one obtained in the frequency-domain in (20) is assessed in Appendix A.

\subsection{Energy analysis}

One advantage of the time-domain formulation is the possibility to perform an energy analysis, which is under study in this section. In the original microstructured configuration, a bounded domain $D=\cup_{i} D_{i} \cup D_{m}$ is considered in order to define the elastic energy. Accordingly, in the homogenized problem, the elastic energy has to be defined in the bounded domain $D \backslash D_{a}$ that excludes the enlarged interface of width $a$, see Figure 4. In this configuration, a term $\mathcal{E}_{a}$ will be associated to the jump conditions at the effective interface. We expect it to account for the bulk energy of the microstructured region of thickness $e$ in the original problem, so that $\mathcal{E}_{a} \geq 0$ is expected.

Multiplying the first equation of (37) by $\Sigma^{h}$, the second by $V^{h}$, then after summation and integration over $D \backslash D_{a}$ one obtains:

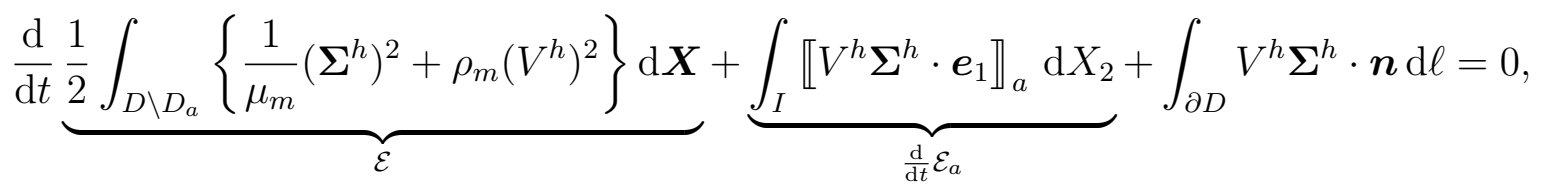




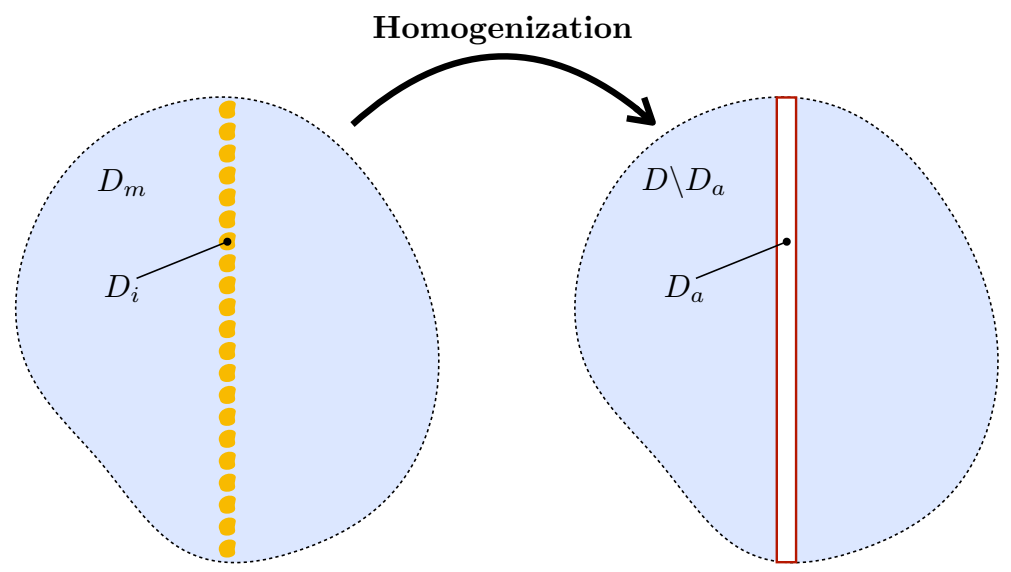

Figure 4: (left) Domain $D=\cup_{i} D_{i} \cup D_{m}$ considered for the energy analysis in the original microstructured configuration, (right) domain $D \backslash D_{a}$ for the analysis of the effective problem.

where the interval $I$ is defined as $I=\left\{X_{2}: \boldsymbol{X} \in D_{a}\right\}$ and $\boldsymbol{n}$ is the inward unit normal on $\partial D$. $\mathcal{E}$ and $\mathcal{E}_{a}$ are respectively some bulk and interface energy terms. The third term involves the velocity and the normal component of the stress vector on the boundaries of the domain $D$. We assume that these wavefields have a compact support so that this third term vanishes if $D$ is chosen large enough.

Focusing on the term $\mathcal{E}_{a}$, then using the relation $\llbracket f g \rrbracket_{a}=\llbracket f \rrbracket_{a}\langle g\rangle_{a}+\llbracket g \rrbracket_{a}\langle f\rangle_{a}$ together with the jump conditions of (37) one obtains

$$
\begin{aligned}
\frac{\mathrm{d}}{\mathrm{d} t} \mathcal{E}_{a} & =\int_{I} h\left\{B\left\langle\frac{\partial V^{h}}{\partial X_{1}}\right\rangle_{a}+B_{2}\left\langle\frac{\partial V^{h}}{\partial X_{2}}\right\rangle_{a}\right\}\left\langle\Sigma_{1}^{h}\right\rangle_{a} \mathrm{~d} X_{2} \\
& +\int_{I} h\left\{S\left\langle\frac{\partial \Sigma_{1}^{h}}{\partial X_{1}}\right\rangle_{a}+C_{1}\left\langle\frac{\partial \Sigma_{1}^{h}}{\partial X_{2}}\right\rangle_{a}+C_{2}\left\langle\frac{\partial \Sigma_{2}^{h}}{\partial X_{2}}\right\rangle_{a}\right\}\left\langle V^{h}\right\rangle_{a} \mathrm{~d} X_{2}+J
\end{aligned}
$$

where one has defined

$$
J=h \rho_{i} \int_{I}\left\{\int_{\Omega_{i}} \frac{\partial W_{i}}{\partial t}\left(\boldsymbol{y}, X_{2}, t\right) \mathrm{d} \boldsymbol{y}\right\}\left\langle V^{h}\right\rangle_{a} \mathrm{~d} X_{2} .
$$

The first two relations of (37) and integration by parts lead to

$$
\begin{aligned}
\frac{\mathrm{d}}{\mathrm{d} t} \mathcal{E}_{a} & =\beta+\frac{h}{2} \frac{\mathrm{d}}{\mathrm{d} t} \int_{I}\left\{S \rho_{m}\left\langle V^{h}\right\rangle_{a}^{2}+\frac{B}{\mu_{m}}\left\langle\Sigma_{1}^{h}\right\rangle_{a}^{2}+\frac{S-C_{2}}{\mu_{m}}\left\langle\Sigma_{2}^{h}\right\rangle_{a}^{2}\right\} \mathrm{d} X_{2} \\
& +h\left(B_{2}-C_{1}\right) \int_{I}\left\langle\frac{\partial V^{h}}{\partial X_{2}}\right\rangle_{a}\left\langle\Sigma_{1}^{h}\right\rangle_{a}+J
\end{aligned}
$$

where $\beta$ is a boundary term on $\partial I$.

Adapting the proof of Appendix A in (16) with an integration in the domain $\Omega \backslash \Omega_{i}$, it is straightforward to show from (21) that one has

$$
B_{2}=C_{1}=\int_{\Omega \backslash \Omega_{i}} \nabla_{\boldsymbol{y}} \Phi^{(1)} \cdot \nabla_{\boldsymbol{y}} \Phi^{(2)} \mathrm{d} \boldsymbol{y} .
$$

The term involving $\mathcal{E}_{a}$ reduces to

$$
\frac{\mathrm{d}}{\mathrm{d} t} \mathcal{E}_{a}=\beta+\frac{h}{2} \frac{\mathrm{d}}{\mathrm{d} t} \int_{I}\left\{S \rho_{m}\left\langle V^{h}\right\rangle_{a}^{2}+\frac{B}{\mu_{m}}\left\langle\Sigma_{1}^{h}\right\rangle_{a}^{2}+\frac{S-C_{2}}{\mu_{m}}\left\langle\Sigma_{2}^{h}\right\rangle_{a}^{2}\right\} \mathrm{d} X_{2}+J
$$


Next, we want to express $J$ in (40) as a quadratic form. To do so, we use the field equations and the boundary conditions in (38) to rewrite $J$ as follows:

$$
\begin{aligned}
J & =-\int_{I} \int_{\partial \Omega_{i}} \boldsymbol{\Sigma}_{i}\left(\boldsymbol{y}, X_{2}, t\right) \cdot \boldsymbol{n}\left\langle V^{h}\left(\cdot, X_{2}, t\right)\right\rangle_{a} \mathrm{~d} \boldsymbol{y} \mathrm{d} X_{2}, \\
& =\int_{I} \int_{\Omega_{i}}\left\{W_{i} \operatorname{div}_{\boldsymbol{y}} \boldsymbol{\Sigma}_{i}+\boldsymbol{\Sigma}_{i} \cdot \boldsymbol{\nabla}_{\boldsymbol{y}} W_{i}\right\} \mathrm{d} \boldsymbol{y} \mathrm{d} X_{2},
\end{aligned}
$$

from which one gets

$$
J=\frac{1}{2} \frac{\mathrm{d}}{\mathrm{d} t} \int_{I} \int_{\Omega_{i}}\left\{h \rho_{i} W_{i}^{2}+\frac{h}{\mu_{i}}\left|\boldsymbol{\Sigma}_{i}\right|^{2}\right\} \mathrm{d} \boldsymbol{y} \mathrm{d} X_{2} .
$$

Finally, the interface term (41) is recast as

$$
\begin{aligned}
\frac{\mathrm{d}}{\mathrm{d} t} \mathcal{E}_{a} & =\beta+\frac{h}{2} \frac{\mathrm{d}}{\mathrm{d} t} \int_{I}\left\{S \rho_{m}\left\langle V^{h}\right\rangle_{a}^{2}+\frac{B}{\mu_{m}}\left\langle\Sigma_{1}^{h}\right\rangle_{a}^{2}+\frac{S-C_{2}}{\mu_{m}}\left\langle\Sigma_{2}^{h}\right\rangle_{a}^{2}\right\} \mathrm{d} X_{2} \\
& +\frac{h}{2} \frac{\mathrm{d}}{\mathrm{d} t} \int_{I} \int_{\Omega_{i}}\left\{\rho_{i} W_{i}^{2}+\frac{1}{\mu_{i}}\left|\boldsymbol{\Sigma}_{i}\right|^{2}\right\} \mathrm{d} \boldsymbol{y} \mathrm{d} X_{2} .
\end{aligned}
$$

To conclude, the bounded domain $D$ is chosen sufficiently large so that the remaining boundary terms on $\partial D$ in (39) and $\beta$ on $\partial I$ in (42) vanish. The identity (39) finally reads as the following conservation equation:

$$
\frac{\mathrm{d}}{\mathrm{d} t}\left(\mathcal{E}+\mathcal{E}_{a}\right)=0
$$

with $\mathcal{E}$ defined in (39) and

$$
\mathcal{E}_{a}=\frac{h}{2} \int_{I}\left\{S \rho_{m}\left\langle V^{h}\right\rangle_{a}^{2}+\frac{B}{\mu_{m}}\left\langle\Sigma_{1}^{h}\right\rangle_{a}^{2}+\frac{S-C_{2}}{\mu_{m}}\left\langle\Sigma_{2}^{h}\right\rangle_{a}^{2}+\int_{\Omega_{i}}\left(\rho_{i} W_{i}^{2}+\frac{1}{\mu_{i}}\left|\Sigma_{i}\right|^{2}\right) \mathrm{d} \boldsymbol{y}\right\} \mathrm{d} X_{2} .
$$

Lastly, the term $\mathcal{E}_{a}$ has to be positive to represent an energy. In this case, (43) is a conservation equation for the total energy $\left(\mathcal{E}+\mathcal{E}_{a}\right)$, a sufficient condition for the continuous problem considered to be stable. Since $\mathcal{E}_{a}$ is a quadratic form, a sufficient condition for $\mathcal{E}_{a} \geq 0$ is $B \geq 0, S \geq 0$ and $\left(S-C_{2}\right) \geq 0$. These three terms can be bounded below as follows:

- $S \geq 0$ if and only if $a / e \geq \varphi$.

- $B \geq 0$. This condition has been proved in the 3D case in (19) when $a=e$. For completeness, it is proved for all $a$ in the 2D case in Appendix B.

- $\left(S-C_{2}\right) \geq 0$ if $a \geq e$. This condition has been proved in the 3D case in (19) when $a=e$. For completeness, it is proved for all $a$ in the 2D case in Appendix C.

To sum up, provided that $a \geq e$ then $\mathcal{E}_{a}$ can be defined as an energy for all $0<\varphi<1$, which is associated with the effective interface of finite thickness $a$. In turn, Equation (43) ensures that the homogenized problem (37) is stable. 


\section{Numerical experiments}

\subsection{Configuration and numerical methods}

\subsubsection{Microstructured configuration}

We consider the propagation of waves across a straight and $h$-periodic row of elliptic inclusions aligned with the $X_{2}$-axis and placed at $X_{1}=0 \mathrm{~m}$. One sets $h=2 \mathrm{~m}$ and the ellipses are defined with semi-major axis $R_{1}=0.8 \mathrm{~m}$, semi-minor axis $R_{2}=0.5 \mathrm{~m}$ and tilt angle $\theta=40^{\circ}$ with respect to the $X_{1}$-axis, which results in a row thickness $e=1.3838 \mathrm{~m}$. The inclusions are highly contrasted, with shear modulus $\mu_{i}=10^{-2} \mu_{m}=10^{8} \mathrm{~kg} \cdot \mathrm{m}^{-1} \cdot \mathrm{s}^{-2}$ while their mass density is such that $\rho_{i}=\rho_{m}=$ $4.44 \cdot 10^{3} \mathrm{~kg} \cdot \mathrm{m}^{-3}$. The corresponding wave velocities are $c_{m}=1500 \mathrm{~m} \cdot \mathrm{s}^{-1}$ and $c_{i}=150 \mathrm{~m} \cdot \mathrm{s}^{-1}$.

A numerical approximation of the solution $\boldsymbol{U}=(V, \boldsymbol{\Sigma})^{\top}$ in this microstructured configuration is obtained using the numerical method presented in (12). The system (1) is discretized on a Cartesian grid and solved using the ADER-4 scheme $(23 ; 13)$. As expected, the mesh size has to be much smaller than $h$ for the inclusions geometry to be suitably approximated, which in turn implies high numerical costs in terms of computation time and memory requirements. Here, the interfaces $\partial \Omega_{i}$ are discretized using the Explicit Simplified Interface Method (ESIM).

\subsubsection{Homogenized model}

In the equivalent homogenized model (37), and owing to the analysis of Section 2.2, the interface thickness is chosen as $a=e$. The effective parameter $S$ in (36) is calculated from the physical parameter values while the parameters $B_{1}, B_{2}, C_{1}$ and $C_{2}$ are computed numerically. Introducing the functional spaces

$$
L_{\mathrm{loc}}^{p}\left(\Omega \backslash \Omega_{i}\right)=\left\{f \in L^{p}\left(K \backslash \Omega_{i}\right) \text { with } K=\right]-\infty, \infty[\times[a, b] \text { for all } a \leq b \text { such that } K \subset \Omega\},
$$

and

$$
\begin{aligned}
\mathcal{H}_{0, j}^{\mathrm{per}}\left(\Omega \backslash \Omega_{i}\right)=\left\{\frac{\partial f}{\partial y_{j}}\right. & \in L_{\mathrm{loc}}^{1}\left(\Omega \backslash \Omega_{i}\right), \frac{\partial f}{\partial y_{k}} \in L_{\mathrm{loc}}^{2}\left(\Omega \backslash \Omega_{i}\right) \text { for } k \neq j, \\
& \text { and } \left.\tilde{\Phi}\left(y_{1}, y_{2}+1\right)=\tilde{\Phi}\left(y_{1}, y_{2}\right) \text { for all } \boldsymbol{y} \in \Omega \backslash \Omega_{i} \text { with } \int_{\Omega \backslash \Omega_{i}} \tilde{\Phi}(\boldsymbol{y}) \mathrm{d} \boldsymbol{y}=0\right\},
\end{aligned}
$$

then the variational formulation associated with (21) reads for all $\tilde{\Phi} \in \mathcal{H}_{0, j}^{\mathrm{per}}\left(\Omega \backslash \Omega_{i}\right)$ :

$$
\int_{\Omega \backslash \Omega_{i}}\left[\boldsymbol{\nabla}_{\boldsymbol{y}} \Phi^{(j)}(\boldsymbol{y})+\boldsymbol{e}_{j}\right] \cdot \boldsymbol{\nabla}_{\boldsymbol{y}} \tilde{\Phi}(\boldsymbol{y}) \mathrm{d} \boldsymbol{y}-\lim _{y_{1} \rightarrow+\infty} \int_{-1 / 2}^{1 / 2}\left[\tilde{\Phi}\left(y_{1}, y_{2}\right)-\tilde{\Phi}\left(-y_{1}, y_{2}\right)\right] \boldsymbol{e}_{1} \cdot \boldsymbol{e}_{j} \mathrm{~d} y_{2}=0 .
$$

The solutions $\Phi^{(j)}$ are then approximated in a bounded domain $\Omega^{b} \backslash \Omega_{i}$, where $\Omega^{b}=\left[-y^{b}, y^{b}\right] \times\left[-\frac{1}{2}, \frac{1}{2}\right]$ and $y^{b}$ is chosen to be sufficiently large with $y^{b}=5$. These approximations are computed using the finite elements method on a single cell. Once these two cell problems are solved numerically, then the four coefficients $B_{1}, B_{2}, C_{1}$ and $C_{2}$ are approximated based on (22) and (30). For the configuration considered, the obtained values are reported in Table 1. Finally, the solution $\boldsymbol{U}^{h}=\left(V^{h}, \boldsymbol{\Sigma}^{h}\right)^{\top}$ of the time-domain effective problem (37) is discretized with a mesh size $\Delta X$ and a time step $\Delta t$ and we denote by $\left(\mathbf{U}^{h}\right)_{i, j}^{n}$ the approximation of $\boldsymbol{U}^{h}$ at point $(i \Delta X, j \Delta X)$ and time $t_{n}=n \Delta t$. A specific numerical method has been developed in (25) to handle the resonant jump conditions considered, which are non-local in time. The latter relies on the introduction of a set of auxiliary variables, locally along the interface, to derive some equivalent jump conditions that are local in time. The resulting 
Table 1: Numerical values of the effective interface parameters featured in (37).

\begin{tabular}{|c|c|c|c|c|c|}
\hline$B_{1}$ & $B$ & $B_{2}$ & $S$ & $C_{1}$ & $C_{2}$ \\
\hline 0.911 & 1.603 & -0.142 & 0.378 & -0.142 & 0.2 \\
\hline
\end{tabular}

system is in turn discretized on a Cartesian grid and solved using the ADER-4 scheme while the geometry of the interface is handled using an extension of the ESIM to the resonant case. We refer the reader to this reference for a detailed account on it.

In the proposed numerical method, the auxiliary variables, which are introduced to derive a local-intime system, rely on the expression of $D\left(k_{m}\right)$ in (A.8). The expression of this coefficient is detailed in Appendix A. The values $\left(\alpha_{r}, k_{r}\right)$ are obtained by solving numerically the eigenvalue problem (A.6). The first four modes $P_{r}$ of the eigenvalue problem (A.6) are shown in Figure 5 for the elliptic inclusions case considered. Referring to the discussion in Appendix A, then the modes $P_{1}$ and $P_{4}$ in Figure 5a and $5 \mathrm{~d}$ have non-null mean values and are linked to resonances at $k_{1}$ and $k_{4}$ in $D\left(k_{m}\right)$. The modes $P_{2}$ and $P_{3}$ represented in Figures $5 \mathrm{~b}$ and $5 \mathrm{c}$ have null mean values and the associated resonances $k_{2}$ and $k_{3}$ are not taken into account in the effective model. Numerically, the infinite sum in $D\left(k_{m}\right)$ is truncated to the first eight resonances associated with modes that have non-null mean values. Computations have been performed to check that the resonances not taken into account have a negligible influence on the effective solution. The numerical values of the parameters $\alpha_{0}$ and $\left(\alpha_{r}, k_{r}\right)$ for $\alpha_{r} \neq 0$, which are used numerically, are reported in Table 2.

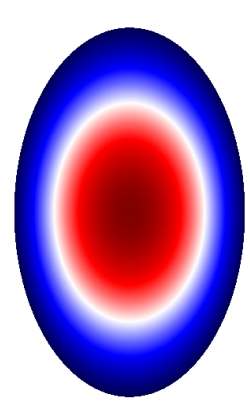

(a) $P_{1}$

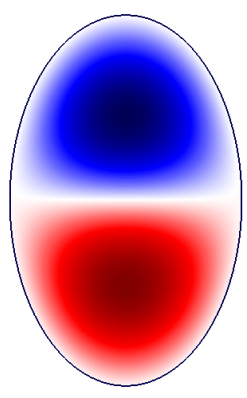

(b) $P_{2}$

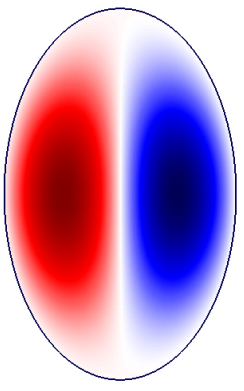

(c) $P_{3}$

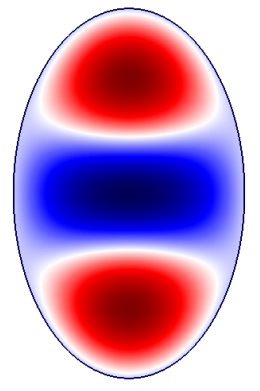

(d) $P_{4}$

Figure 5: First four modes $P_{r}$ of the eigenvalue problem (A.6) for the elliptic inclusions considered.

Table 2: Resonances parameters in (A.8).

\begin{tabular}{|c|c|c|c|c|c|c|c|c|c|}
\hline$r$ & 0 & 1 & 4 & 8 & 9 & 13 & 16 & 21 & 23 \\
\hline$\alpha_{r}$ & 0.314 & 0.462 & 0.144 & 0.148 & 0.069 & 0.078 & 0.037 & 0.053 & 0.081 \\
\hline$k_{r}\left(\mathrm{rad} \cdot \mathrm{m}^{-1}\right)$ & & 0.4 & 0.734 & 1.015 & 1.091 & 1.308 & 1.452 & 1.625 & 1.642 \\
\hline
\end{tabular}

\subsubsection{Initial condition and forcing}

To design the initial conditions or the forcing term, the following source function is introduced

$$
F(\xi)= \begin{cases}\sum_{k=0}^{3} \beta_{k} \sin \left(2^{k} k_{m} \xi\right) & \text { if }-\frac{c_{m}}{f_{0}} \leq \xi \leq 0 \\ 0 & \text { otherwise, }\end{cases}
$$


with $f_{0}$ the central frequency, $\beta_{0}=1, \beta_{1}=-21 / 32, \beta_{2}=63 / 768$, and $\beta_{3}=-1 / 512$, which entails that $F$ is of class $C^{6}$. Figure 6 displays the Fourier transform $\hat{F}$ of $F$ as a function of $f=k c_{m} /(2 \pi)$ for $f_{0} \in\{25,50,100\} \mathrm{Hz}$. The small parameter corresponding to these central frequencies reads $\eta\left(f_{0}\right) \in\{0.21,0.42,0.84\}$, respectively. While in Fig. 6 the dashed line indicates the central frequency $f_{0}$, it should be noted that the frequency content of $\hat{F}$ extends to frequencies much higher than $f_{0}$ thus resulting in higher values of the parameter $\eta$ for the associated wavelengths. In this figure, the blue crosses denote the resonant frequencies $f_{r}=k_{r} c_{m} /(2 \pi)$ in $D\left(k_{m}\right)$ defined in (A.8) and associated with modes with non-zero mean (resonant frequencies with zero-mean modes are not displayed here).

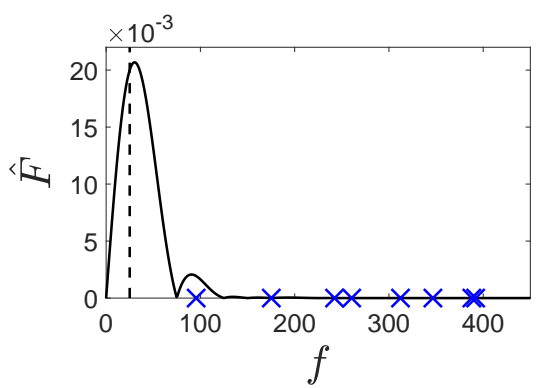

(a) $f_{0}=25 \mathrm{~Hz}$

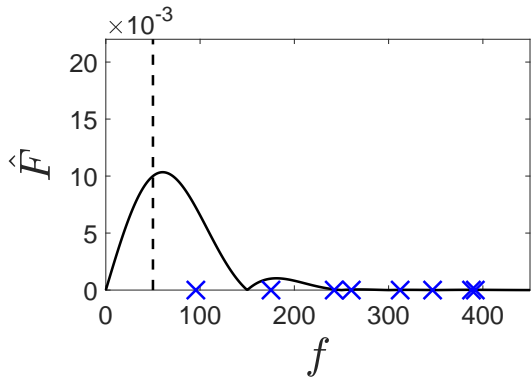

(b) $f_{0}=50 \mathrm{~Hz}$

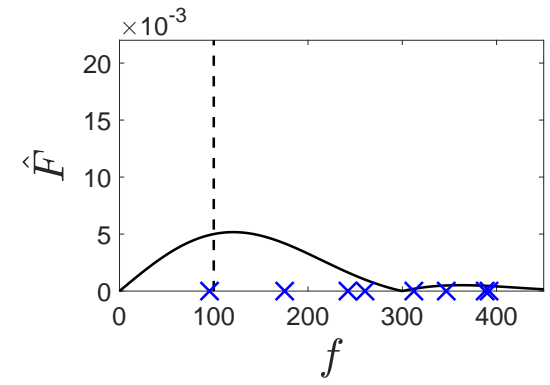

(c) $f_{0}=100 \mathrm{~Hz}$

Figure 6: Fourier transform $\hat{F}$ of the source function for the three different values $f_{0}$ considered (here indicated by the dashed lines). The blue crosses denote the frequencies associated with resonances $k_{r}$, see (A.8), of non-zero mean modes.

Incident plane wave. In a first case, an incident plane wave is considered and the initial conditions read

$$
\boldsymbol{U}(\boldsymbol{X}, 0)=\left(\begin{array}{c}
\frac{1}{\mu_{m}} \\
-\frac{1}{c_{m}} \\
0
\end{array}\right) F\left(\left(\boldsymbol{X}-\boldsymbol{X}_{I}\right) \cdot \boldsymbol{e}_{1}\right)
$$

where the initialization point $\boldsymbol{X}_{I}=(-2,0) \mathrm{m}$ is chosen such that compactly supported initial conditions (47) do not intersect the enlarged interface. The initial velocity field and profile are displayed in Figure 7 for $f_{0}=100 \mathrm{~Hz}$. The velocity profile is normalized by the maximum homogenized velocity. From now on, it will be the case for each velocity profile displayed.

Point source. In a second case, we consider a point source located at $\boldsymbol{X}_{S}=(-35,0) \mathrm{m}$ and such that (1) reads:

$$
\left\{\begin{array}{l}
\frac{\partial \boldsymbol{\Sigma}}{\partial t}(\boldsymbol{X}, t)=\mu_{m} \boldsymbol{\nabla} V(\boldsymbol{X}, t) \\
\rho_{m} \frac{\partial V}{\partial t}(\boldsymbol{X}, t)=\operatorname{div} \boldsymbol{\Sigma}(\boldsymbol{X}, t)+\rho_{m} \delta\left(\boldsymbol{X}-\boldsymbol{X}_{s}\right) F\left(t c_{m}\right),
\end{array}\right.
$$

with $\delta\left(\boldsymbol{X}-\boldsymbol{X}_{s}\right)$ being a Dirac delta function at $\boldsymbol{X}=\boldsymbol{X}_{s}$.

\subsubsection{Numerical errors}

To assess whether the homogenized model provides a satisfying approximation of the original problem in the microstructured configuration, we compare the corresponding numerical solutions. 


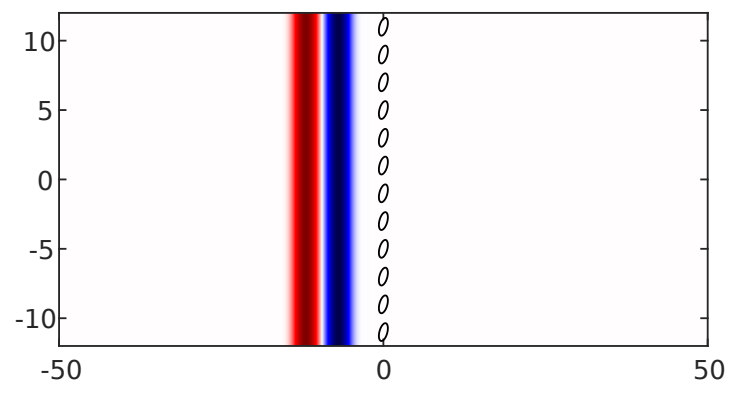

(a) Waveform

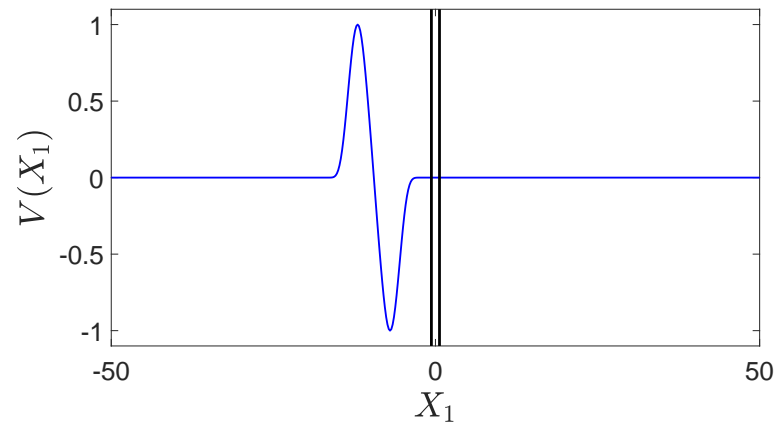

(b) $X_{1}$-profile at $X_{2}=-1 \mathrm{~m}$

Figure 7: Velocity field at the time $t=0$ for the incident plane wave given by (47) with $f_{0}=100 \mathrm{~Hz}$.

With $\boldsymbol{U}$ and $\boldsymbol{U}^{h}$ being respectively the exact solution of the original problem in the microstructured configuration and in the homogenized model, we denote by $\mathbf{U}$ and $\mathbf{U}^{h}$ their numerical approximations. Defining the global modeling error $\varepsilon_{T}=\left\|\boldsymbol{U}-\boldsymbol{U}^{h}\right\|$, then one has formally by triangular inequality:

$$
\varepsilon_{T} \leq \underbrace{\|\boldsymbol{U}-\mathbf{U}\|}_{\varepsilon_{1}}+\underbrace{\left\|\mathbf{U}-\mathbf{U}^{h}\right\|}_{\varepsilon_{2}}+\underbrace{\left\|\mathbf{U}^{h}-\boldsymbol{U}^{h}\right\|}_{\varepsilon_{3}} .
$$

The term $\varepsilon_{1}$ is the numerical error associated with the simulation in the microstructured configuration while $\varepsilon_{3}$ is the one associated with the simulation based on the homogenized model. Both are governed and controlled by the numerical methods employed, see (11) and (25), respectively. In appropriate implementations of the latter, these errors are considered to be negligible compared to $\varepsilon_{2}$. As a consequence, we consider that the numerical error $\varepsilon_{2}=\left\|\mathbf{U}-\mathbf{U}^{h}\right\|$ provides a reliable estimation of the global modeling error $\varepsilon_{T}$ and can be used to measure the validity of the homogenized model.

The homogenized model of Section 2 is derived under Assumptions 1, i.e., with asymptotics being performed in the limit $\eta \rightarrow 0$ while it is assumed that $\mu_{i} / \mu_{m}=\mathcal{O}\left(\eta^{2}\right)$ to preserve resonances. In practice, we apply this model to a given geometric and material configuration for which the parameters $h, \mu_{i}$ and $\mu_{m}$ are set. The signal (46) is also considered as a source term and the latter carries a range of wavelengths $\lambda$ that are in turn associated with a parameter $\eta(\lambda)=2 \pi h / \lambda=2 \pi h f / c_{m}$. For Assumptions 1 to hold then one must have both $\eta(\lambda) \ll 1$ and $\eta(\lambda)=\mathcal{O}\left(\sqrt{\mu_{i} / \mu_{m}}\right)$.

As a consequence, the agreement between simulations for the microstructured and the homogenized problems are expected to deteriorate as $\eta(\lambda)$ approaches 1 . For the source signal (46) considered, we will only focus on $\eta\left(f_{0}\right)$ but, as seen in Figure 6, the frequency content of this source extends to higher frequencies for which the associated wavelengths may lie beyond the validity domain of Assumptions 1.

\subsection{Incident plane wave at normal incidence}

In a first example, we consider an incident plane wave at normal incidence, see Section 3.1.3. The velocity profiles of the numerical solutions in the original microstructured configuration are presented in Figure 8a for different mesh sizes. Figure 8b compares the profiles of velocity for the homogenized model, computed either numerically on a grid of mesh size $\Delta X=0.2 \mathrm{~m}$ or semi-analytically. The latter computation is performed using the method described in (25) which relies on the calculation of the reflexion and transmission coefficients from (A.4). On the one hand, the numerical solution 


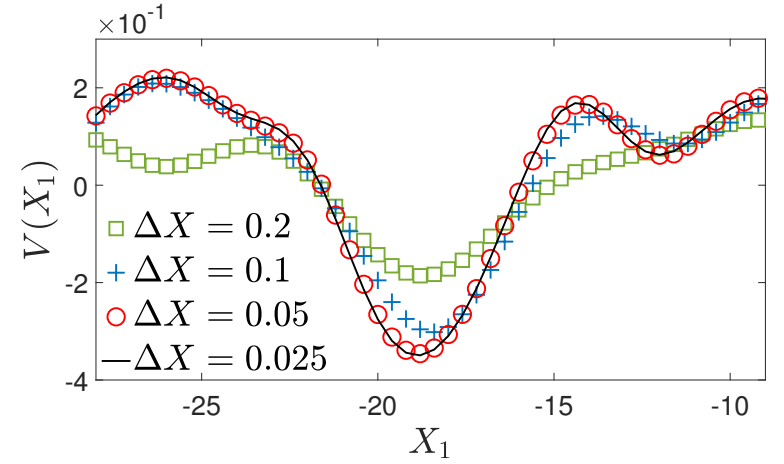

(a) Microstructured configuration: numerical solutions

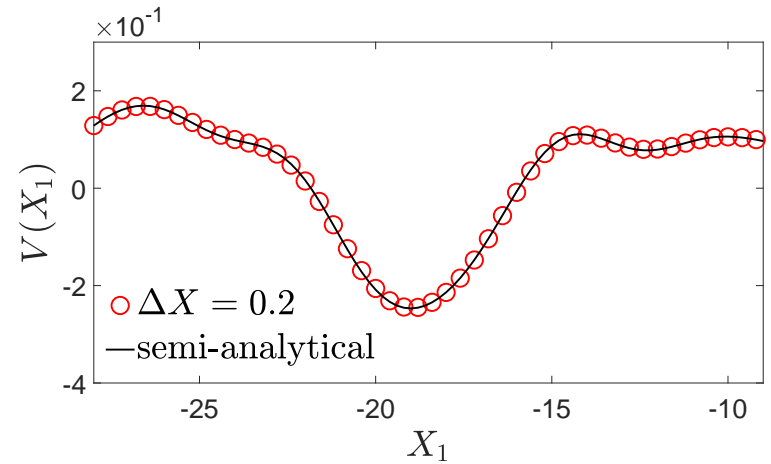

(b) Homogenized model: numerical and semi-analytical solutions

Figure 8: Velocity profiles for the microstructured configuration and using the homogenized model. The source term is such that $f_{0}=100 \mathrm{~Hz}$.

for the microstructured configuration on the fine grid $\Delta X=0.025 \mathrm{~m}$ is assumed to have converged, see Fig. 8a. On the other hand, a coarse grid $\Delta X=0.2 \mathrm{~m}$ suffices when using the homogenized model, see Fig. 8b. As an indication of the numerical gain, at $f_{0}=100 \mathrm{~Hz}$, a computational time of 47 seconds for the homogenized model ( $\Delta X=0.2 \mathrm{~m}$ in Fig. $8 \mathrm{~b}$ ) corresponds to a computational time of 4.8 hours for the original microstructured configuration $(\Delta X=0.025 \mathrm{~m}$ in Fig. 8a). The extra time needed for the original problem is due to the smaller mesh size but also to the smaller time step imposed by the CFL condition for numerical stability. These two mesh sizes will be used in numerical experiments from now on.

The velocity fields for the microstructured configuration and for the homogenized model together with their respective profiles along $X_{2}=-1 \mathrm{~m}$ are reported in Figure 9 at time $t=31.7 \mathrm{~ms}$ for $f_{0} \in\{25,50,100\} \mathrm{Hz}$. The corresponding discrepancies between both solutions is measured in the $L^{2}$-norm for $X_{1} \in[-50 ;-5] \mathrm{m}$ and at $X_{2}=-1 \mathrm{~m}$. The relative errors are of about $4 \%, 7 \%$ and $10 \%$, respectively. For the stress component $\Sigma_{1}$, these errors are of about $3 \%, 7 \%$ and $13 \%$. Since the errors measurements are comparable for the velocity and stress fields, we will now evaluate the agreement between the homogenized model and the microstructured problem only in terms of the velocity field. As expected from the discussion in Section 3.1.4, the solutions agree at low frequency but deviate from one another as the parameter $\eta\left(f_{0}\right)$ increases to 1 and does not conform to the shear modulus ratio. Finally, in Figure 9, it can be observed that the dispersive nature of the material is amplified, in both the microstructured configuration and in the homogenized model, as $f_{0}$ increases. This behavior reflects the fact that resonances play an increasing role. At low frequency, the observed wavefield is almost comparable to the case of a non-resonant interface (with non-constrasted inclusions), see (10), while at higher frequencies some energy is still radiated by the interface after the incident wave has crossed the latter.

In the case $f_{0}=50 \mathrm{~Hz}$, the velocity in the microstructured and homogenized configuration is recorded from $t=0$ to $t=475 \mathrm{~ms}$ at one receiver. This receiver is located on the right of the enlarged interface at $\boldsymbol{X}_{T}=\left(X_{T}, 0\right) \mathrm{m}$, with $X_{T}=10 \mathrm{~m}$. From these data, the Fourier transform of the transmitted velocity $\hat{V}\left(\boldsymbol{X}_{T}, f\right)$ is computed and its logarithm is displayed in Figure 10. The dashed line indicates the logarithm of the Fourier transform of the incident wave. The three logarithms are normalized by the maximum of the logarithm of the homogenized Fourier transform. Around the resonances for the microstructured configuration, we can see within a small frequency band a high transmission followed 

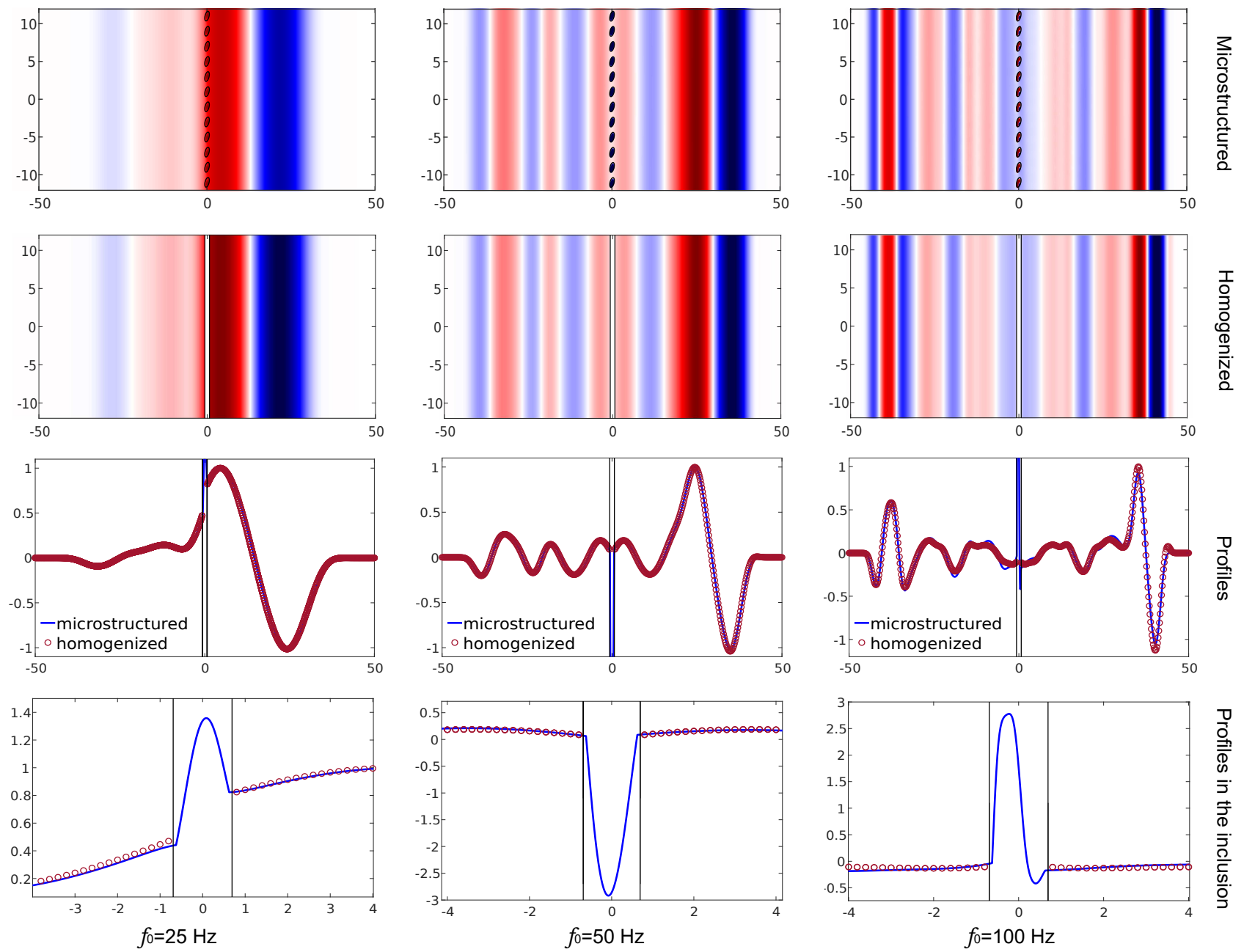

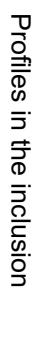

Figure 9: (Top two rows) velocity fields for the microstructured configuration and for the homogenized model for an illumination by an incident plane wave at normal incidence. (Third row) comparison of the corresponding velocity profiles at $X_{2}=-1 \mathrm{~m}$. (Bottom row) zoom in the inclusion. (left) $f_{0}=25 \mathrm{~Hz}$ so that $\eta\left(f_{0}\right)=0.21$, (center) $f_{0}=50 \mathrm{~Hz}$ so that $\eta\left(f_{0}\right)=0.42$, and (right) $f_{0}=100 \mathrm{~Hz}$ so that $\eta\left(f_{0}\right)=0.84$.

by a high reflexion. A zoom on the first two resonances is also displayed. The first one is associated with a mode of non-null mean value, see Figure 5a, while the mode of the second one has a null mean value, see Figure 5b. Around the first resonance taken into account in $D\left(k_{m}\right)$ (A.8), we have a good agreement between both Fourier transforms. On the contrary, the behaviour of the solution is not well described by the homogenized solution around the second resonance. The same holds for each resonance associated with a mode of null mean value. However, these missed resonant frequencies also correspond to values of $\eta$ which are around or higher than 1 . Thus, even if the behaviour of the solution is not well described, it is in any case for a range of frequencies that go beyond the low-frequency hypothesis of homogenization. Furthermore, it can also be noticed that these missed resonant frequencies are not solicited a lot by the incident velocity. Therefore, it can be assumed that it does not induce a much greater error.

\subsection{Point source}

In the previous example of Section 3.2, the $X_{2}$-invariance of the illumination considered implies that the $X_{2}$-derivatives vanish in the effective model (37) so that the associated homogenized 


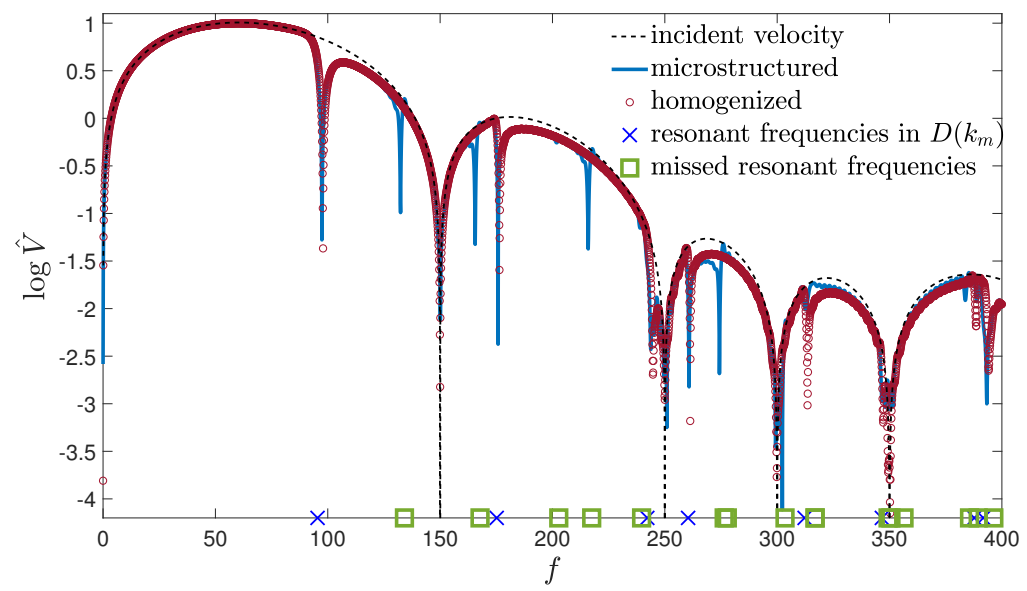

(a)

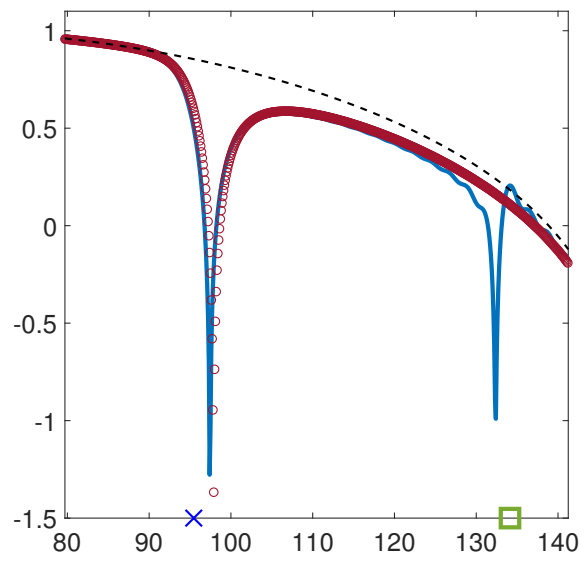

(b)

Figure 10: (a) Logarithm of the Fourier transform $\hat{V}\left(\boldsymbol{X}_{T}, f\right)$ for the microstructured and the homogenized configuration. The dashed line indicates the logarithm of the Fourier transform of the incident velocity. The blue crosses denote the resonant frequencies taken into account in $D\left(k_{m}\right)$ (A.8) and the green squares denote the missed resonant frequencies of zero mean modes. (b) Zoom on the first two resonances.

coefficients $B_{2}, C_{1}$ and $C_{2}$ play no role. As a consequence, to deal with a full $2 \mathrm{D}$ configuration, we consider an illumination by the point source described in (48). The physical and numerical parameters are the same as in the previous example. Moreover, the computational domain is defined as $[-70 \mathrm{~m} ; 35 \mathrm{~m}] \times[-132 \mathrm{~m} ; 132 \mathrm{~m}]$, i.e. it is chosen large enough along the $X_{2}$-axis to avoid reflections from the top and bottom boundaries while Perfectly Matched Layers are used in the background domain on the left and right boundaries.

The velocity fields for the microstructured configuration and for the homogenized model are displayed at times $t \in\left\{t_{1}, t_{2}, t_{3}, t_{4}\right\}=\{25.3,38.0,50.7,63.3\} \mathrm{ms}$ on the figures 11,12 and 13 , which correspond to a source central frequency $f_{0} \in\{25,50,100\} \mathrm{Hz}$, respectively. The $X_{1}$-profiles of both solutions at $X_{2}=1 \mathrm{~m}$ are compared on Figure 14. Quantitatively, the discrepancies between solutions are again measured in the $L^{2}$-norm for $X_{1} \in[-50 \mathrm{~m} ;-5 \mathrm{~m}]$ and at $X_{2}=1 \mathrm{~m}$. The associated relative errors are of about $2 \%, 9 \%$ and $13 \%$, respectively, which is of same order than the ones obtained in Section 3.2.

As in the previous example, the agreement between solutions deteriorates as $\eta$ approaches 1 . Figure 14 also displays the velocity profiles zoomed in within a single inclusion in the microstructured configuration. As the frequency increases then shorter wavelengths are trapped within the inclusion and more resonant frequencies featured in (A.8) are solicited, thereby explaining the discrepancies between the two models.

\section{Conclusion}

This study has focused on the propagation of waves across a periodic row of highly-contrasted inclusions, a problem which has been tackled by a homogenization technique. More specifically, the homogenization process has been performed directly in the time domain. The high-contrast between the inclusions and the matrix amounts in a scaling of the shear moduli as $\eta^{2}$ where $\eta$ is the usual small parameter corresponding to the long-wavelength regime. As a consequence, this produces wavelengths within the inclusions that are comparable to the size of the latter. The specific homoge- 

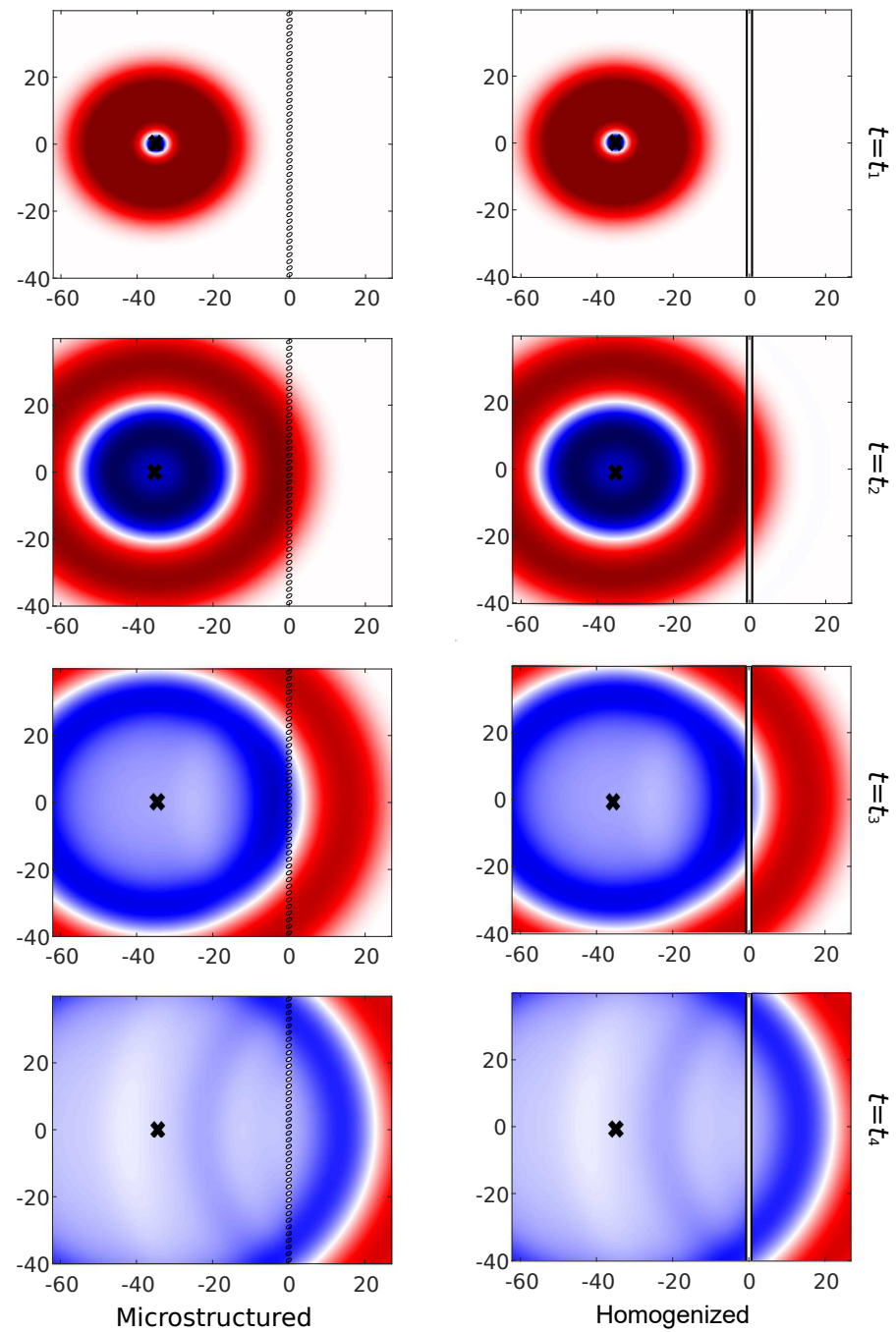

Figure 11: (Left) velocity fields for the microstructured configuration and (right) for the homogenized model for $f_{0}=25 \mathrm{~Hz}$ (so that $\eta\left(f_{0}\right)=0.21$ ) and at different times $t \in\{25.3,38.0,50.7,63.3\} \mathrm{ms}$. A point source is located at $(-35,0) \mathrm{m}$ and symbolized by a black cross. Only a subset of the computational domain is shown here.

nization process has led to effective jump conditions that are (i) non-local in time and (ii) apply on the boundaries of an equivalent enlarged interface. One notes that no calculation need to be performed anymore within this region. Having the thickness of the effective interface larger than the width of the original microstructured array has been shown to be a sufficient condition to have a stable effective problem. The correspondence of this time-domain effective model with the frequency-dependent jump conditions developed in (20) has also been established. Finally, numerical examples have been provided to illustrate the model obtained and to compare its solutions with simulations involving the original microstructure. A good agreement has been found at low excitation frequency and it has been discussed how it deteriorates as the frequency increases.

Perspectives include the derivation of an effective model at higher order, as discussed in (9;20), to account for the resonances associated with modes with zero mean, which are missed in the current model. Moreover, it is important to assess to what extent these resonances play a role for the problem under consideration. The extension of this work to the full three dimensional elasticity case is also 
of interest and the derivation of the corresponding effective model should not pose major technical difficulties. Another possible future direction concerns the design of meta-interfaces through a dedicated topological optimization process for microstructured periodic arrays in order to reach some objective effective properties, see the approach developed in (6) for bulk metamaterials. For example, this would be of interest for noise reduction by thin resonant meta-interfaces, see $(14 ; 22)$.

Acknowledgements. We acknowledge the stimulating exchanges promoted by the GDR MecaWave of the French Centre National de la Recherche Scientifique. CB, BL and MT are thankful to Rémi Cornaggia for fruitful discussions.

\section{Conflict of interest}

The authors declare that they have no conflict of interest.

\section{Appendix A. Frequency-domain formulation}

With the effective model (37) being derived in the time domain, this section focuses on assessing its equivalence with the frequency-domain formulation obtained in (20). First, the frequency Fourier transform is defined:

$$
\mathscr{F}[f](\boldsymbol{X}, \omega)=\hat{f}(\boldsymbol{X}, \omega)=\int_{\mathbb{R}} f(\boldsymbol{X}, t) e^{-i \omega t} \mathrm{~d} t,
$$

along with the convolution product:

$$
[f * g](t)=\int_{\mathbb{R}} f\left(t-t^{\prime}\right) g(t) \mathrm{d} t^{\prime}
$$

Due to the final effective jump condition (37), we seek closed-form identity for the field $W_{i}$ solution of (38). To do so, let us consider the time-domain Green's function associated with the inclusion domain $\Omega_{i}$ and a source point $\boldsymbol{y}_{0}$, i.e. the field $G:(\boldsymbol{y}, t) \mapsto G\left(\boldsymbol{y}, \boldsymbol{y}_{0}, t\right)$ that is the fundamental solution of the problem:

$$
\begin{cases}\frac{\partial^{2} G}{\partial t^{2}}\left(\boldsymbol{y}, \boldsymbol{y}_{0}, t\right)-\frac{\mu_{i}}{\rho_{i} h^{2}} \Delta_{y} G\left(\boldsymbol{y}, \boldsymbol{y}_{0}, t\right)=\delta\left(\boldsymbol{y}-\boldsymbol{y}_{0}\right) \delta(t) & \left(\boldsymbol{y} \in \Omega_{i}\right), \\ G\left(\boldsymbol{y}, \boldsymbol{y}_{0}, t\right)=0 & \left(\boldsymbol{y} \in \partial \Omega_{i}\right), \\ G\left(\boldsymbol{y}, \boldsymbol{y}_{0}, 0\right)=\frac{\partial G}{\partial t}\left(\boldsymbol{y}, \boldsymbol{y}_{0}, 0\right)=0 & \left(\boldsymbol{y} \in \Omega_{i}\right) .\end{cases}
$$

The equations (38) at time $\left(t-t^{\prime}\right)$ are multiplied by $G$ taken at time $t^{\prime}$ and integrated on $\Omega_{i} \times[0, t]$, which leads to

$$
\int_{\Omega_{i}}\left\{\left[\frac{\partial^{2} W_{i}}{\partial t^{2}} * G\right](t)-\frac{\mu_{i}}{\rho_{i} h^{2}}\left[\Delta_{\boldsymbol{y}} W_{i} * G\right](t)\right\} \mathrm{d} \boldsymbol{y}=0 .
$$

Integrating by parts twice, the first term in time and the second term in space, respectively, and using the boundary conditions for $W_{i}$ and $G$ yields:

$$
W_{i}\left(\boldsymbol{y}_{0}, X_{2}, t\right)=-\frac{\mu_{i}}{\rho_{i} h^{2}}\left[\left\langle V^{h}\left(\cdot, X_{2}, \cdot\right)\right\rangle_{a} * \int_{\partial \Omega_{i}} \nabla_{\boldsymbol{y}} G\left(\boldsymbol{y}, \boldsymbol{y}_{0}, \cdot\right) \cdot \boldsymbol{n} \mathrm{d} \ell\right](t) .
$$

Going back to (37), we now get formally in the frequency domain:

$\mathscr{F}\left[\rho_{i} \int_{\Omega_{i}} \frac{\partial W_{i}}{\partial t} \mathrm{~d} \boldsymbol{y}\right](\boldsymbol{X}, \omega)=\frac{\rho_{i}}{\rho_{m}}\left\langle\operatorname{div} \hat{\boldsymbol{\Sigma}}^{h}\left(\cdot, X_{2}, \omega\right)\right\rangle_{a} \int_{\Omega_{i}}\left\{-\frac{\mu_{i}}{\rho_{i} h^{2}} \int_{\partial \Omega_{i}} \nabla_{\boldsymbol{y}_{0}} \hat{G}\left(\boldsymbol{y}, \boldsymbol{y}_{0}, \omega\right) \cdot \boldsymbol{n} \mathrm{d} \ell_{0}\right\} \mathrm{d} \boldsymbol{y}$, 
where we have used the identity $i \omega \rho_{m} \hat{V}^{h}=\operatorname{div} \hat{\Sigma}^{h}$ and the fact that $\hat{G}\left(\boldsymbol{y}, \boldsymbol{y}_{0}, \omega\right)=\hat{G}\left(\boldsymbol{y}_{0}, \boldsymbol{y}, \omega\right)$. We define the field $\hat{V}_{k_{m}}$ as

$$
\hat{V}_{k_{m}}(\boldsymbol{y}, \omega)=-\frac{\mu_{i}}{\rho_{i} h^{2}} \int_{\partial \Omega_{i}} \nabla_{\boldsymbol{y}_{\mathbf{0}}} \hat{G}\left(\boldsymbol{y}, \boldsymbol{y}_{0}, \omega\right) \cdot \boldsymbol{n} \mathrm{d} \ell_{0} .
$$

Owing to the following relation satisfied by $G$ in the frequency domain

$$
\Delta_{\boldsymbol{y}} \hat{G}\left(\boldsymbol{y}, \boldsymbol{y}_{0}, \omega\right)+\frac{\rho_{i} \mu_{m}}{\rho_{m} \mu_{0}} \hat{G}\left(\boldsymbol{y}, \boldsymbol{y}_{0}, \omega\right)=-\frac{\rho_{i} h^{2}}{\mu_{i}} \delta\left(\boldsymbol{y}-\boldsymbol{y}_{0}\right)
$$

then it turns out that $\hat{V}_{k_{m}}$ is the solution of the following problem:

$$
\begin{cases}\Delta_{\boldsymbol{y}} \hat{V}_{k_{m}}(\boldsymbol{y}, \omega)+\kappa\left(k_{m}\right)^{2} \hat{V}_{k_{m}}(\boldsymbol{y}, \omega)=0 & \left(\boldsymbol{y} \in \Omega_{i}\right), \\ \hat{V}_{k_{m}}(\boldsymbol{y}, \omega)=1 & \left(\boldsymbol{y} \in \partial \Omega_{i}\right),\end{cases}
$$

where one has defined $\kappa\left(k_{m}\right)=k_{m} h \sqrt{\frac{\rho_{i} \mu_{m}}{\rho_{m} \mu_{i}}}$.

Therefore, provided that the Fourier transforms are well-defined, the time-domain model developed here is in agreement with the frequency-domain model of (20), see Equation (44) in the latter:

$$
\begin{cases}\llbracket \hat{V}^{h} \rrbracket_{a}=h\left\{B\left\langle\frac{\partial \hat{V}^{h}}{\partial X_{1}}\right\rangle_{a}+B_{2}\left\langle\frac{\partial \hat{V}^{h}}{\partial X_{2}}\right\rangle_{a}\right\} & \left(X_{2} \in \mathbb{R}\right) \\ \llbracket \hat{\Sigma}_{1}^{h} \rrbracket_{a}=h\left\{S\left\langle\frac{\partial \hat{\Sigma}_{1}^{h}}{\partial X_{1}}\right\rangle_{a}+C_{1}\left\langle\frac{\partial \hat{\Sigma}_{1}^{h}}{\partial X_{2}}\right\rangle_{a}+C_{2}\left\langle\frac{\partial \hat{\Sigma}_{2}^{h}}{\partial X_{2}}\right\rangle_{a}+D\left(k_{m}\right)\left\langle\operatorname{div} \hat{\Sigma}^{h}\right\rangle_{a}\right\} & \left(X_{2} \in \mathbb{R}\right),\end{cases}
$$

where, due to (A.1) and (A.2), one has:

$$
D\left(k_{m}\right)=\frac{\rho_{i}}{\rho_{m}} \int_{\Omega_{i}} \hat{V}_{k_{m}}(\boldsymbol{y}) \mathrm{d} \boldsymbol{y}
$$

Consequently, the agreement of the time-domain effective model with the frequency-dependent jump conditions is established. However, thanks to the time-domain formulation, we were able to perform an energy analysis and provide a sufficient condition for the effective model to be stable.

As shown in (20), the solution $\hat{V}_{k_{m}}$ to (A.3) can be found as an expansion onto the function basis of the eigensystem $\left(\lambda_{r}, P_{r}\right)_{r \geq 1}$ that is associated with the following self-adjoint eigenvalue problem within the inclusion:

$$
\begin{cases}\Delta_{\boldsymbol{y}} P_{r}(\boldsymbol{y})+\lambda_{r} P_{r}(\boldsymbol{y})=0 & \text { in } \Omega_{i} \\ P_{r}(\boldsymbol{y})=0 & \text { on } \partial \Omega_{i} .\end{cases}
$$

In particular, let us define the resonant frequencies $\left\{k_{r}\right\}_{r \geq 1}$ and the real-valued coefficients $\left\{\alpha_{r}\right\}_{r \geq 0}$ as follows

$$
\alpha_{0}=\int_{\Omega_{i}} \mathrm{~d} \boldsymbol{y} \quad \alpha_{r}=\int_{\Omega_{i}} P_{r}(\boldsymbol{y}) \mathrm{d} \boldsymbol{y} \quad \text { and } \quad k_{r}=\frac{1}{h} \sqrt{\frac{\rho_{m} \mu_{i}}{\rho_{i} \mu_{m}} \lambda_{r}} .
$$


Then the term $D\left(k_{m}\right)$ in (A.5) can be recast as the following infinite series:

$$
D\left(k_{m}\right)=\frac{\rho_{i}}{\rho_{m}}\left\{\alpha_{0}-\sum_{r \geq 1} \alpha_{r}^{2} \frac{k_{m}^{2}}{k_{m}^{2}-k_{r}^{2}}\right\} .
$$

Note that, due to the expression of $\alpha_{r}$ in (A.7), the eigenmodes $P_{r}$ that have zero mean value do not contribute to the effective model obtained. Moreover, this expression of $D\left(k_{m}\right)$ will be used later on in the numerical method chosen to handle this non-local in time jump conditions.

\section{Appendix B. Positivity of the parameter $B$}

We seek a lower bound for the parameter $B$ that is defined by (36) and (22). It is useful for the energy analysis of Section 2.2, see Equation (44). To do so, we consider the variational formulation (45) that is satisfied by the field $\Phi^{(1)}$. Moreover, let us define the following quadratic functional:

$$
\mathcal{L}(\tilde{\Phi})=\int_{\Omega \backslash \Omega_{i}}\left[\frac{1}{2} \boldsymbol{\nabla}_{\boldsymbol{y}} \tilde{\Phi}(\boldsymbol{y})+\boldsymbol{e}_{1}\right] \cdot \nabla_{\boldsymbol{y}} \tilde{\Phi}(\boldsymbol{y}) \mathrm{d} \boldsymbol{y}-\lim _{y_{1} \rightarrow+\infty} \int_{-1 / 2}^{1 / 2}\left[\tilde{\Phi}\left(y_{1}, y_{2}\right)-\tilde{\Phi}\left(-y_{1}, y_{2}\right)\right] \mathrm{d} y_{2} .
$$

Then, owing to (45), the field $\Phi^{(1)}$ minimizes $\mathcal{L}$ on $\mathcal{H}_{0,1}^{\text {per }}\left(\Omega \backslash \Omega_{i}\right)$ and one has

$$
\mathcal{L}\left(\Phi^{(1)}\right)=-\frac{B_{1}}{2}+\frac{1}{2} \int_{\Omega \backslash \Omega_{i}} \nabla_{\boldsymbol{y}} \Phi^{(1)} \cdot \boldsymbol{e}_{1} \mathrm{~d} \boldsymbol{y} .
$$

To have an explicit expression of the second term, we multiply the cell problem (21) by the function $y_{1}$ and we integrate in the bounded domain $\Omega^{b} \backslash \Omega_{i}$, i.e.

$$
\int_{\Omega^{b} \backslash \Omega_{i}} \Delta_{\boldsymbol{y}}\left(\Phi^{(1)}+y_{1}\right) y_{1} \mathrm{~d} \boldsymbol{y}=0
$$

By integration by parts and due to the periodicity and boundary conditions for $\Phi^{(1)}$, this equation leads to

$$
\int_{-1 / 2}^{1 / 2}\left[\boldsymbol{\nabla}_{\boldsymbol{y}} \Phi^{(1)}\left(-y_{1}^{b}, y_{2}\right)+\boldsymbol{\nabla}_{\boldsymbol{y}} \Phi^{(1)}\left(y_{1}^{b}, y_{2}\right)\right] \cdot y_{1}^{b} \boldsymbol{e}_{1} \mathrm{~d} y_{2}+2 y_{1}^{b}-\int_{\Omega^{b} \backslash \Omega_{i}} \boldsymbol{\nabla}_{\boldsymbol{y}} \Phi^{(1)} \cdot \boldsymbol{e}_{1} \mathrm{~d} \boldsymbol{y}-\int_{\Omega^{b} \backslash \Omega_{i}} \mathrm{~d} \boldsymbol{y}=0 .
$$

Given that the last integral is equal to $\left(2 y_{1}^{b}-e \varphi / h\right)$, then considering the previous identity in the limit $y_{1}^{b} \rightarrow+\infty$ entails

$$
\int_{\Omega \backslash \Omega_{i}} \nabla_{\boldsymbol{y}} \Phi^{(1)} \cdot \boldsymbol{e}_{1} \mathrm{~d} \boldsymbol{y}=\frac{e \varphi}{h}
$$

and thus, from (B.1), one gets

$$
\mathcal{L}\left(\Phi^{(1)}\right)=-\frac{B_{1}}{2}+\frac{e \varphi}{2 h} .
$$

As a consequence and due to the minimization principle for $\mathcal{L}$, one obtains the following lower bound for $B_{1}$ :

$$
B_{1} \geq \frac{e \varphi}{h}-2 \mathcal{L}(\tilde{\Phi}) \quad \forall \tilde{\Phi} \in \mathcal{H}_{0,1}^{\text {per }}\left(\Omega \backslash \Omega_{i}\right)
$$

To have an explicit bound, we define $\tilde{\Phi} \in \mathcal{H}_{0,1}^{\text {per }}\left(\Omega \backslash \Omega_{i}\right)$ as the piecewise linear function:

$$
\tilde{\Phi}(\boldsymbol{y})=2 \tilde{\beta} h \frac{y_{1}}{e} \quad \text { if } 0 \leq\left|y_{1}\right| \leq \frac{e}{2 h} \quad \text { and } \quad \tilde{\Phi}(\boldsymbol{y})=\tilde{\beta} \operatorname{sign}\left(y_{1}\right) \quad \text { if }\left|y_{1}\right| \geq \frac{e}{2 h}
$$


with $\tilde{\beta}$ a constant, from which one gets:

$$
\mathcal{L}(\tilde{\Phi})=2 \frac{h}{e}(1-\varphi) \tilde{\beta}^{2}-2 \tilde{\beta} \varphi .
$$

As a quadratic function of $\tilde{\beta}$, then $\mathcal{L}(\tilde{\Phi})$ reaches a minimum for $\tilde{\beta}=\frac{e \varphi}{2 h(1-\varphi)}$, which inserted in (B.2) yields:

$$
B_{1} \geq \frac{e \varphi}{h}+\frac{e \varphi^{2}}{h(1-\varphi)} .
$$

To conclude, using (36), one finally obtains the following positive lower bound for $B$ :

$$
B \geq \frac{a}{h}+\frac{e \varphi}{h}+\frac{e \varphi^{2}}{h(1-\varphi)} \geq 0 .
$$

\section{Appendix C. Positivity of the term $\left(S-C_{2}\right)$}

We seek a lower bound for the term $\left(S-C_{2}\right)$ that is featured in (44) and is defined by (36) and (30). Let us introduce $\Psi^{(2)}=\left(\nabla_{\boldsymbol{y}} \Phi^{(2)}+\boldsymbol{e}_{2}\right)$. Owing to the definition of the field $\Phi^{(2)}$ as the solution to (21) and based on the definition of the functional space $\mathcal{H}_{0,2}^{\text {per }}\left(\Omega \backslash \Omega_{i}\right)$ we consider in addition the following admissibility space:

$$
\begin{aligned}
\mathcal{W}^{\text {per }}\left(\Omega \backslash \Omega_{i}\right)=\left\{\left(\boldsymbol{f}-\boldsymbol{e}_{2}\right) \in L_{\mathrm{loc}}^{2}\left(\Omega \backslash \Omega_{i}\right) \text { with } \boldsymbol{f}\left(y_{1}, y_{2}+1\right)=\boldsymbol{f}\left(y_{1}, y_{2}\right) \text { for all } \boldsymbol{y} \in \Omega \backslash \Omega_{i}\right. \\
\text { and such that } \left.\operatorname{div}_{\boldsymbol{y}} \boldsymbol{f}=0 \text { in } \Omega \backslash \Omega_{i}, \boldsymbol{f} \cdot \boldsymbol{n}=0 \text { on } \partial \Omega_{i} \text { and } \lim _{y_{1} \rightarrow \pm \infty} \boldsymbol{f}=\boldsymbol{e}_{2}\right\},
\end{aligned}
$$

so that $\Psi^{(2)} \in \mathcal{W}^{\text {per }}\left(\Omega \backslash \Omega_{i}\right)$. We also introduce for any $\tilde{\Psi} \in \mathcal{W}^{\text {per }}\left(\Omega \backslash \Omega_{i}\right)$ the following functional:

$$
\mathcal{M}(\tilde{\boldsymbol{\Psi}})=\frac{1}{2} \int_{\Omega \backslash \Omega_{i}}\left|\tilde{\boldsymbol{\Psi}}-\boldsymbol{e}_{2}\right|^{2} \mathrm{~d} \boldsymbol{y}
$$

Upon noticing that for any admissible field $\tilde{\Psi}$ the derivative of $\mathcal{M}$ in $\mathcal{W}^{\text {per }}\left(\Omega \backslash \Omega_{i}\right)$ satisfies

$$
\mathcal{M}^{\prime}\left[\boldsymbol{\Psi}^{(2)}\right] \tilde{\boldsymbol{\Psi}}=\int_{\Omega \backslash \Omega_{i}} \nabla_{\boldsymbol{y}} \Phi^{(2)} \cdot \tilde{\boldsymbol{\Psi}} \mathrm{d} \boldsymbol{y}=\int_{\partial\left(\Omega \backslash \Omega_{i}\right)} \Phi^{(2)} \tilde{\boldsymbol{\Psi}} \cdot \boldsymbol{n} \mathrm{d} \boldsymbol{y}-\int_{\Omega \backslash \Omega_{i}} \Phi^{(2)} \operatorname{div}_{\boldsymbol{y}} \tilde{\boldsymbol{\Psi}} \mathrm{d} \boldsymbol{y}=0
$$

then $\Psi^{(2)}$ minimizes the quadratic functional $\mathcal{M}$. Moreover, from the cell problem (21) one has

$$
\int_{\Omega \backslash \Omega_{i}} \Delta_{\boldsymbol{y}}\left(\Phi^{(2)}+y_{2}\right) \Phi^{(2)} \mathrm{d} \boldsymbol{y}=0 .
$$

By integration by parts and due to the periodicity and boundary conditions for $\Phi^{(2)}$ and its derivatives, this leads to

$$
C_{2}=\int_{\Omega \backslash \Omega_{i}}\left|\nabla_{\boldsymbol{y}} \Phi^{(2)}\right|^{2} \mathrm{~d} \boldsymbol{y}=2 \mathcal{M}\left(\Psi^{(2)}\right) .
$$

As a consequence, we obtain that $C_{2} \leq 2 \mathcal{M}(\tilde{\Psi})$ for all $\tilde{\Psi} \in \mathcal{W}^{\text {per }}\left(\Omega \backslash \Omega_{i}\right)$. Finally, we define $\tilde{\Psi}$ as

$$
\tilde{\boldsymbol{\Psi}}(\boldsymbol{y})=\mathbf{0} \quad \text { if } 0 \leq\left|y_{1}\right|<\frac{e}{2 h} \quad \text { and } \quad \tilde{\boldsymbol{\Psi}}(\boldsymbol{y})=\boldsymbol{e}_{2} \quad \text { if }\left|y_{1}\right| \geq \frac{e}{2 h}
$$

from which one gets $\mathcal{M}(\tilde{\Psi})=e(1-\varphi) /(2 h)$. From the minimization principle, we finally obtain the following lower bound $\left(S-C_{2}\right) \geq(a-e) / h$, which proves that $\left(S-C_{2}\right) \geq 0$ if $a \geq e$. 
[1] Auriault, J.L., Bonnet, G.: Dynamique des composites Ãllastiques pÃ lriodiques. Arch Mech. 37(4-5), 269-284 (1985)

[2] Auriault, J.L., Boutin, C.: Long wavelength inner-resonance cut-off frequencies in elastic composite materials. International Journal of Solids and Structures 49(23-24), 3269-3281 (2012). DOI 10.1016/j.ijsolstr.2012.07.002

[3] Bensoussan, A., Lions, J.L., Papanicolaou, G.: Asymptotic analysis for periodic structures. AMS Chelsea Publishing (2011)

[4] Bonnet-Bendhia, A., Drissi, D., Gmati, N.: Simulation of muffler's transmission losses by a homogenized finite element method. Journal of Computational Acoustics 12(3), 447âĂŞ474 (2004)

[5] Bouchitté, G., Bourel, C., Felbacq, D.: Homogenization of the 3d maxwell system near resonances and artificial magnetism. Comptes Rendus Mathematique 347(9-10), 571-576 (2009). DOI 10.1016/j.crma.2009.02.027

[6] Cornaggia, R., Bellis, C.: Tuning effective dynamical properties of periodic media by FFTaccelerated topological optimization. International Journal for Numerical Methods in Engineering (2020). DOI 10.1002/nme.6352

[7] David, M., Pideri, C., Marigo, J.J.: Homogenized interface model describing inhomogeneities located on a surface. Journal of Elasticity 109(2), 153-187 (2012)

[8] Delourme, B.: ModÃ̂́les asymptotiques des interfaces fines et pÃl'riodiques en ÃllectromagnÃltisme. PhD thesis - UniversitÃl' Pierre et Marie Curie - Paris VI (2010)

[9] Felbacq, D., Bouchitté, G.: Theory of mesoscopic magnetism in photonic crystals. Physical Review Letters 94(18) (2005). DOI 10.1103/physrevlett.94.183902

[10] Lombard, B., Maurel, A., Marigo, J.J.: Numerical modeling of the acoustic wave propagation across an homogenized rigid microstructure in the time domain. Journal of Computational Physics 335, 558-577 (2017)

[11] Lombard, B., Piraux, J.: Numerical treatment of two-dimensional interfaces for acoustic and elastic waves. Journal of Computational Physics 195(1), 90-116 (2004). DOI 10.1016/j.jcp. 2003.09.024

[12] Lombard, B., Piraux, J., Gélis, C., Virieux, J.: Free and smooth boundaries in 2-D finitedifference schemes for transient elastic waves. Geophysical Journal International 172(1), 252261 (2008). DOI 10.1111/j.1365-246x.2007.03620.x

[13] Lorcher, F., Munz, C.D.: Lax-wendroff-type schemes of arbitrary order in several space dimensions. IMA Journal of Numerical Analysis 27(3), 593-615 (2006). DOI 10.1093/imanum/drl031

[14] Ma, G., Yang, M., Xiao, S., Yang, Z., Sheng, P.: Acoustic metasurface with hybrid resonances. Nature Materials 13(9), 873-878 (2014). DOI 10.1038/nmat3994

[15] Marigo, J.J., Maurel, A.: Homogenization models for thin rigid structured surfaces and films. The Journal of the Acoustical Society of America 140(1), 260-273 (2016) 
[16] Marigo, J.J., Maurel, A., Pham, K., Sbitti, A.: Effective dynamic properites of a row of elastic inclusions: The case of scalar shear waves. Journal of Elasticity 128(2), 265-289 (2017)

[17] Marigo, J.J., Pideri, C.: The effective behaviour of elastic bodies containing microcracks or microholes localized on a surface. International Journal of Damage Mechanics, SAGE Publications 20, 1151-1177 (2011)

[18] Maurel, A., Mercier, J.F., Pham, K., Marigo, J.J., Ourir, A.: Enhanced resonance of sparse arrays of helmholtz resonators - application to perfect absorption. The Journal of the Acoustical Society of America 145(4), 2552-2560 (2019). DOI 10.1121/1.5098948

[19] Maurel, A., Pham, K., Marigo, J.J.: Homogenization of thin 3d periodic structures in the time domain-effective boundary and jump conditions. In: V. Romero-García, A.C. HladkyHennion (eds.) Fundamentals and Applications of Acoustic Metamaterials. Wiley (2019). DOI $10.1002 / 9781119649182$

[20] Pham, K., Maurel, A., Marigo, J.J.: Two scale homogenization of a row of locally resonant inclusions - the case of shear waves. Journal of the Mechanics and Physics of Solids 106, 80-94 (2017)

[21] Sanchez-Hubert, J., Sanchez-Palencia, E.: Introduction aux méthodes asymptotiques et à l'homogénéisation. Collection Mathématiques Appliquées pour la Maîtrise (1992)

[22] Schwan, L., Umnova, O., Boutin, C.: Sound absorption and reflection from a resonant metasurface: Homogenisation model with experimental validation. Wave Motion 72, 154-172 (2017). DOI 10.1016/j.wavemoti.2017.02.004

[23] Schwartzkopff, T., Dumbser, M., Munz, C.D.: Fast high order ADER schemes for linear hyperbolic equations. Journal of Computational Physics 197(2), 532-539 (2004). DOI 10.1016/j.jcp.2003.12.007

[24] Sheng, P., Zhang, X., Liu, Z., Chan, C.: Locally resonant sonic materials. Physica B: Condensed Matter 338(1-4), 201-205 (2003). DOI 10.1016/s0921-4526(03)00487-3

[25] Touboul, M., Lombard, B., Bellis, C.: Time-domain simulation of wave propagation across resonant meta-interfaces. Journal of Computational Physics 414, 109474 (2020). DOI 10.1016/ j.jcp.2020.109474

[26] Zhikov, V.V.: On an extension of the method of two-scale convergence and its applications. Sbornik: Mathematics 191(7), 973-1014 (2000). DOI 10.1070/sm2000v191n07abeh000491

[27] Zhikov, V.V.: On spectrum gaps of some divergent elliptic operators with periodic coefficients. St. Petersburg Mathematical Journal 16(05), 773-791 (2005). DOI 10.1090/ s1061-0022-05-00878-2 

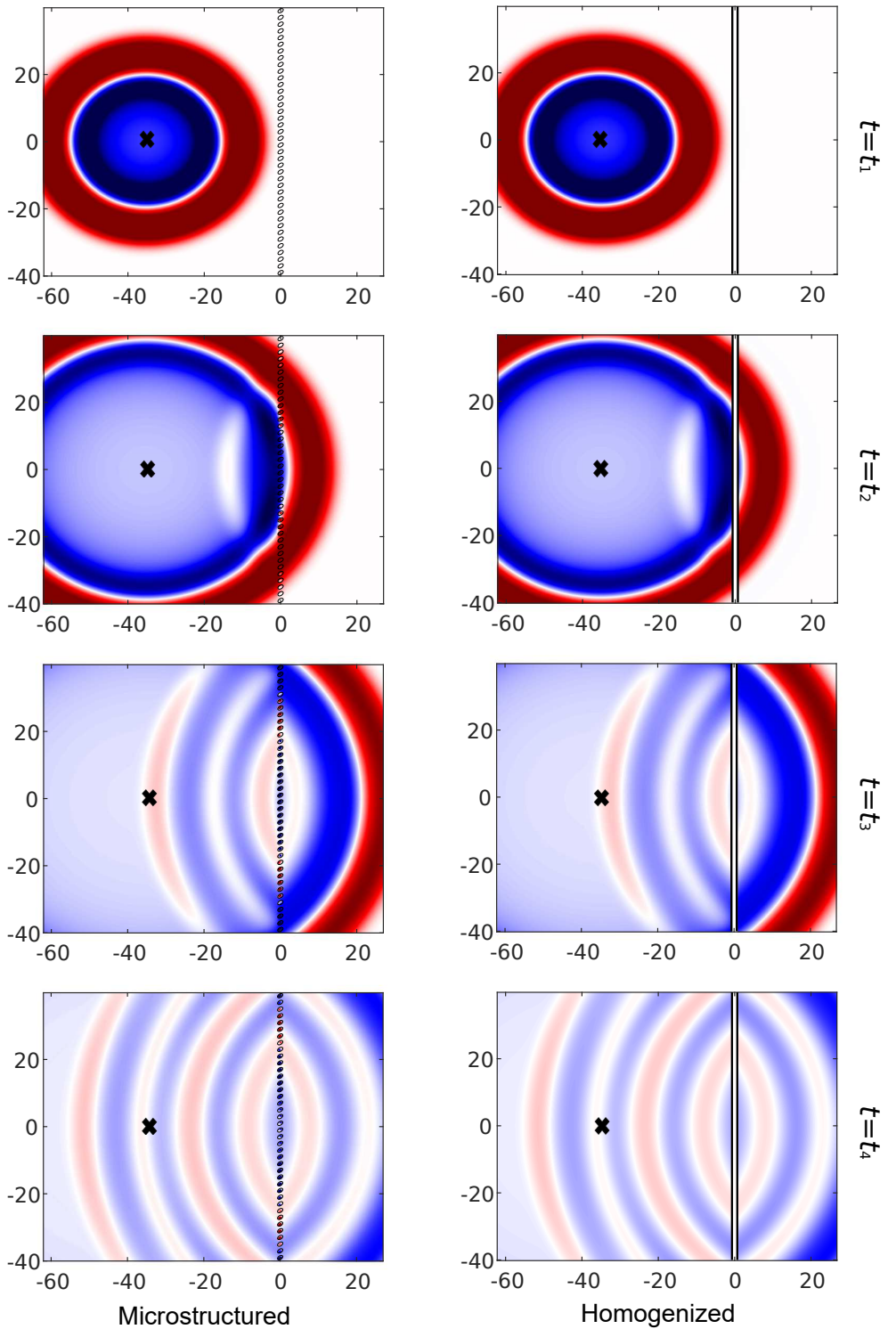

Figure 12: (Left) velocity fields for the microstructured configuration and (right) for the homogenized model for $f_{0}=50 \mathrm{~Hz}$ (so that $\eta\left(f_{0}\right)=0.42$ ) and at different times $t \in\{25.3,38.0,50.7,63.3\} \mathrm{ms}$. A point source is located at $(-35,0) \mathrm{m}$ and symbolized by a black cross. Only a subset of the computational domain is shown here. 

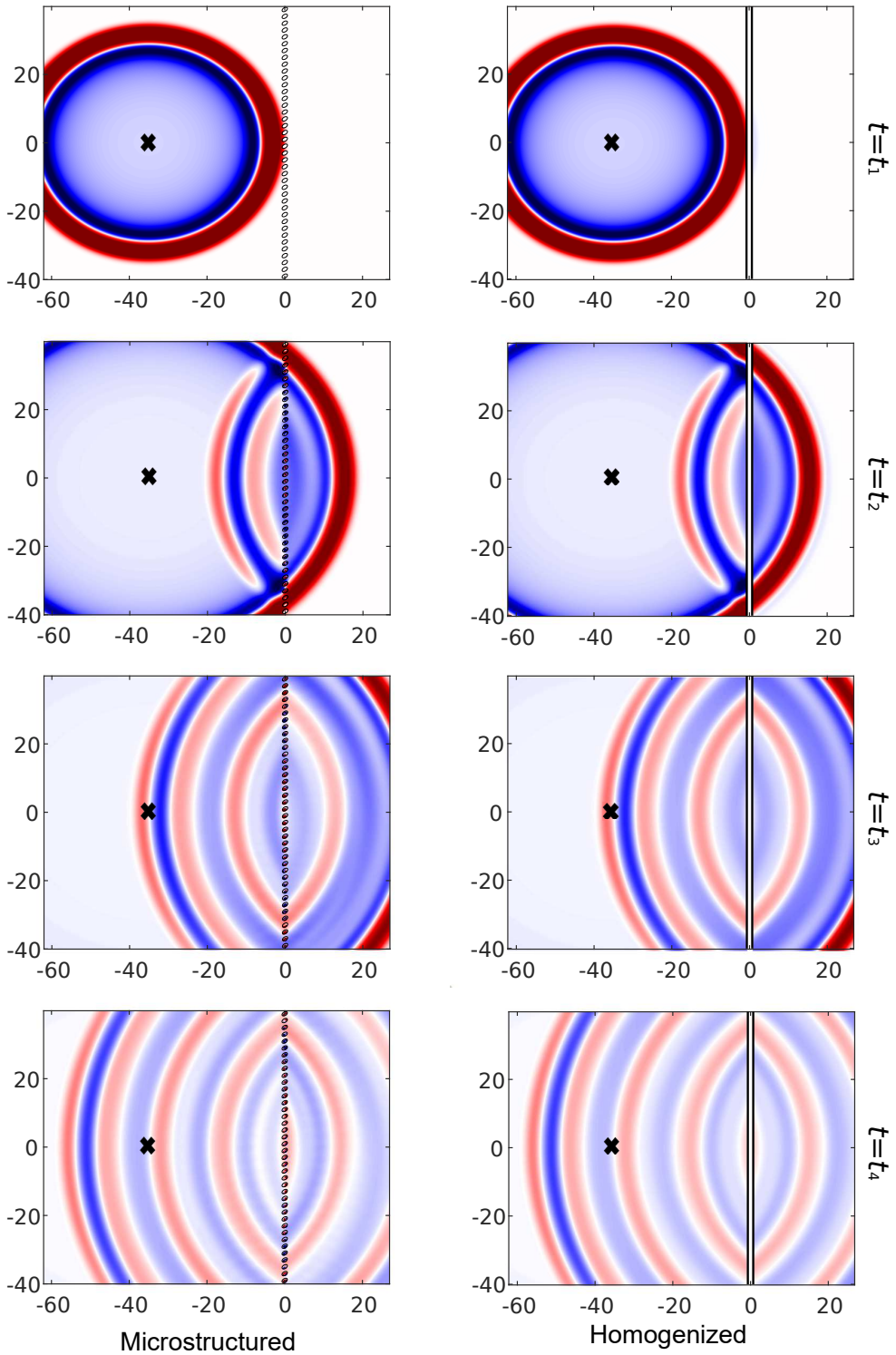

Figure 13: (Left) velocity fields for the microstructured configuration and (right) for the homogenized model for $f_{0}=$ $100 \mathrm{~Hz}$ (so that $\eta\left(f_{0}\right)=0.84$ ) and at different times $t \in\{25.3,38.0,50.7,63.3\} \mathrm{ms}$. A point source is located at $(-35,0) \mathrm{m}$ and symbolized by a black cross. Only a subset of the computational domain is shown here. 

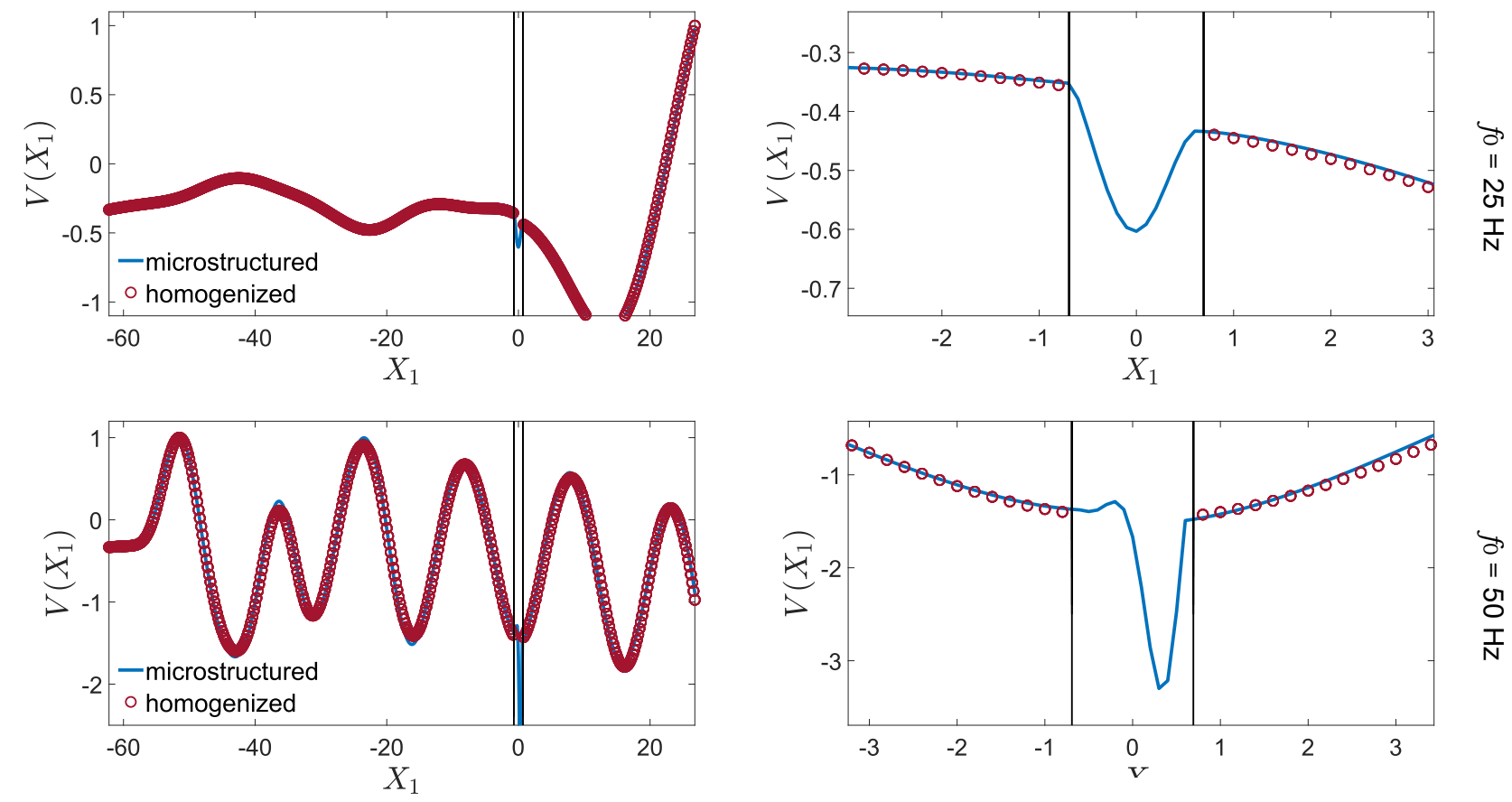

I
11
엉
T

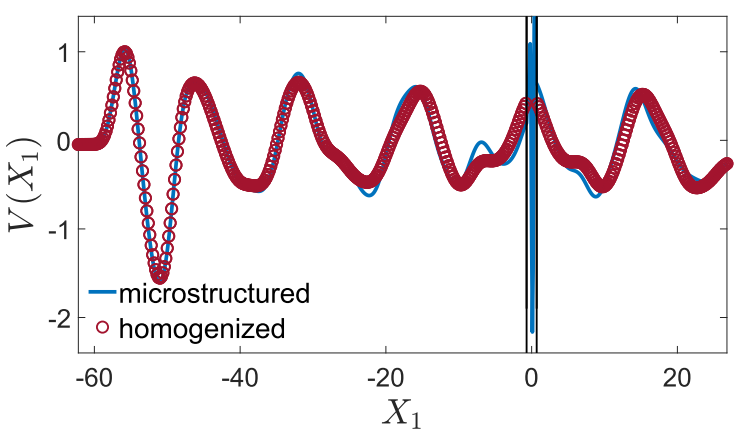

$X_{1}$ profiles of velocity

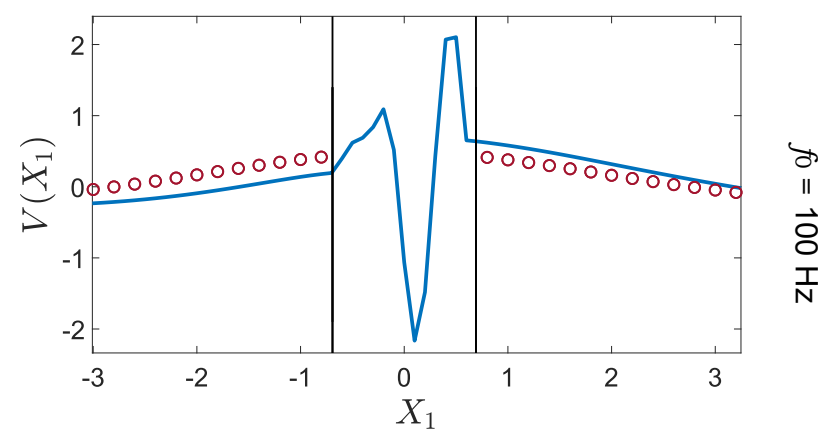

Zoom on the profiles in the inclusion

Figure 14: Comparisons of the velocity profiles for the microstructured configuration and the homogenized model at $X_{2}=1 \mathrm{~m}$. (Left) subset of the computational domain, and (right) zoom in the inclusion region. From top to bottom rows: $f_{0} \in\{25,50,100\} \mathrm{Hz}$, respectively, which corresponds to $\eta\left(f_{0}\right) \in\{0.21,0.42,0.84\}$. 